\title{
Incidence of pests and viral disease on pepino (Solanum muricatum Ait.) in Kanagawa Prefecture, Japan
}

Ok-Kyung Kim, Tadashi Ishikawa§, Yoshihiro Yamada§, Takuma Sato ${ }^{\ddagger}$, Hirosuke Shinohara ${ }^{\ddagger}$, Ken Takahatal

‡ Laboratory of Plant Pathology, Faculty of Agriculture, Tokyo University of Agriculture, Atsugi-shi, Kanagawa, Japan $\S$ Laboratory of Entomology, Faculty of Agriculture, Tokyo University of Agriculture, Atsugi-shi, Kanagawa, Japan

| Laboratory of Vegetables, Faculty of Agriculture, Tokyo University of Agriculture, Atsugi-shi, Kanagawa, Japan

Corresponding author: Tadashi Ishikawa (chuishikawa@gmail.com)

Academic editor: Jenő Kontschán

Received: 05 Jul 2017 | Accepted: 17 Aug 2017 | Published: 22 Aug 2017

Citation: Kim O, Ishikawa T, Yamada Y, Sato T, Shinohara H, Takahata K (2017) Incidence of pests and viral disease on pepino (Solanum muricatum Ait.) in Kanagawa Prefecture, Japan. Biodiversity Data Journal 5: e14879. https://doi.org/10.3897/BDJ.5.e14879

\section{Abstract}

\section{Background}

The solanaceous fruit crop pepino (Solanum muricatum Ait.), originating in the Andes, is grown commercially in South American countries and New Zealand. In these areas, pests and diseases of pepino have been identified well; however, to date, these have seldom been investigated in detail in Japan. Herein, we attempt to reconstruct an agricultural production system for commercial pepino crops in Japan, and evaluate the incidence of pests and viral diseases on pepino. The findings of this study will facilitate in developing a better crop system for the commercial cultivation of healthy pepino fruits. 


\section{New information}

A total of 11 species, comprising nine insects and two mites, were recognized as pests of pepino plants in our experimental fields in Kanagawa Prefecture, central Honshu, Japan. Of these pest species, the two-spotted spider mite Tetranychus urticae Koch, 1836 and the cotton aphid Aphis gossypii Glover, 1877, were remarkably abundant than the other pest species. Eventually, 13 species, including two previously recorded, are currently recognized as the pests of pepino in Japan. With regard to viruses, we tested two species Alfalfa mosaic virus (AMV) and Cucumber mosaic virus (CMV), as well as three genera Carlavirus, Potexvirus, and Potyvirus. No virus was detected in symptomatic pepino leaves collected in our experimental fields. This is a first report on the identification of pests on pepino plants in Kanagawa Prefecture, Japan and elucidates the relationship between currently occurring pests of pepino plants and potential viral pathogens that they can transmit.

\section{Keywords}

insects, mites, virus vector, virus, sweet cucumber

\section{Introduction}

Pepino, the Spanish name for sweet cucumber, (Solanum muricatum Ait.), is a solanaceous plant cultivated as a fruit crop. It originated in the Andes, became popular in several countries and regions of South America (Heiser 1964), and then it was introduced to Central America and New Zealand. In Japan, cultivation of pepino began in 1984 based on pepino fruits imported from New Zealand in 1983 (Sakata 2011). Since then, pepino caused a major boom and its cultivation rapidly spread throughout Japan within a few years. However, the production gradually declined prior to 1990 due to the low soluble solids content (Brix) in the Japanese pepino fruits (less than $8^{\circ}$ Brix) (Sakata 2011). Currently, farmers in Japan do not grow pepino, except for people with gardening as a hobby, who cultivate pepino.

In 2016, our research team began a project for regional development "Launching of Nodaibranded Pepino Crop" conducted by Faculty of Agriculture, Tokyo University of Agriculture (TUA; Nodai is a Japanese abbreviated name of the university). The main purpose of this project was to produce high quality and flavorsome pepino fruits with sufficient soluble solids content. As a recent achievement of this project, Takahata (2017) succeeded in increasing the soluble solids content of pepino fruits by using a washer ring (metal washer) at the bottom of the stem. This technique strongly contributes to improving the quality and flavor of pepino fruits. 
To date, at least 24 species of insect and mite pests on pepino (Larraín 2002) and one virus infected to pepino (Jones et al. 1980) have been recorded in the native range of pepino, the Andes. In contrast, in regions where pepino was introduced other than Japan, such as New Zealand, China and Turkey, there are only a few records on the pests (two species: Galbreath and Clearwater 1983, Akyazi 2012) and viruses (two species: Thomas et al. 1980, Abouelnasr et al. 2014) in the academic literature. In Japan, little has been known in detail about the pests and viruses of pepino, except that inadequately identified pests such as spider mites and aphids damage to pepino.

It is important to establish solid pest control in its commercial cultivation to produce high quality and stable pepinos. Unfortunately, however, no pesticides applicable to pepino plants have been registered in Japan; this could be attributed to the few detailed studies on pests and diseases of pepino. Therefore, our research team has tried to comprehensively elucidate the pests and viral diseases of pepino in this project in order to contribute to the accumulation of basic knowledge toward the establishment of its pest control. This paper documents the results of our field survey on pests and diseases of pepino in Kanagawa Prefecture, central Japan.

\section{Materials and methods}

\section{Study sites}

This study was conducted at the Atsugi Campus (35.432N 139.346E; at altitudes between 25 and 62 meters above sea level) of Tokyo University of Agriculture (TUA), Atsugi City, Kanagawa Prefecture, Japan, which is surrounded by residential quarters and a woody and grassy park (Fig. 1). The total site area of the campus is approximately 17.3 ha, within which several greenhouses and open fields for experimental use are present. The study site is located in a warm-temperate climate zone and has an annual mean temperature of $15.3{ }^{\circ} \mathrm{C}$ and annual mean precipitation of $1,729.9 \mathrm{~mm}$ (Japan Meteorological Agency 2017). In the campus, three survey plots were set (Fig. 1); one of these was an open field (approximately $70 \mathrm{~m}^{2}$ ), where 40 pepino plants were cultivated (Plot A) (Fig. 2); another was a greenhouse (approximately $53 \mathrm{~m}^{2}$ ), in which 60 pot pepino plants were grown (Plot B) (Fig. 3); and the other was also a greenhouse (approximately $90 \mathrm{~m}^{2}$ ), in which approximately 400 pot pepino plants were grown (Plot C) (Fig. 4). In these plots, acaricides were applied approximately every two weeks in order to prevent pepino plants from withering due to mites, except for 20 pepino plants in Plot $A$; when the density of mites became high, appropriate chemicals were sprayed. All the plots were located within a radius of $250 \mathrm{~m}$. 


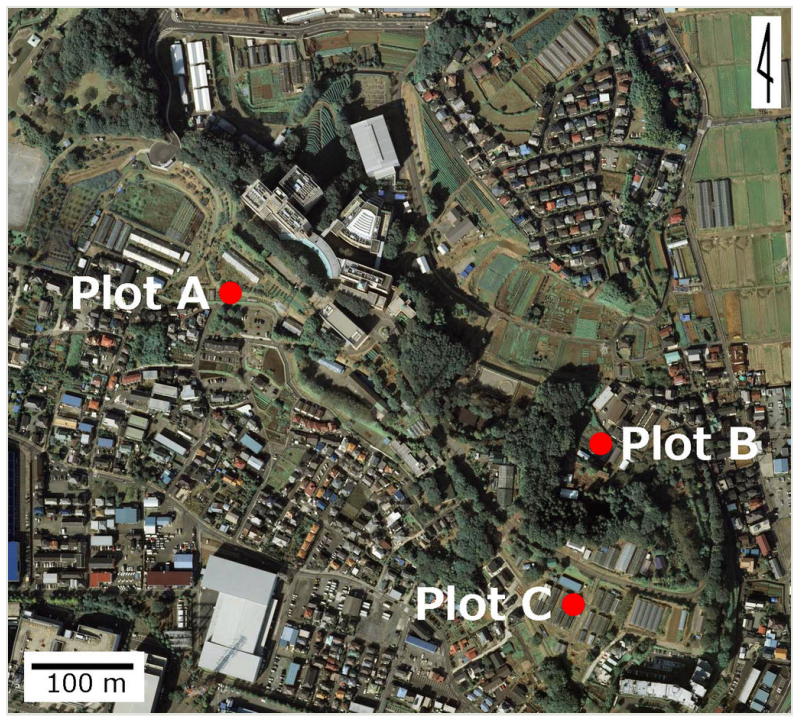

Figure 1. doi

Aerial photograph of the Atsugi Campus of Tokyo University of Agriculture (TUA) and surrounding residential quarters, with locations of the three survey plots (taken in 2007 by the Geospatial Information Authority of Japan).

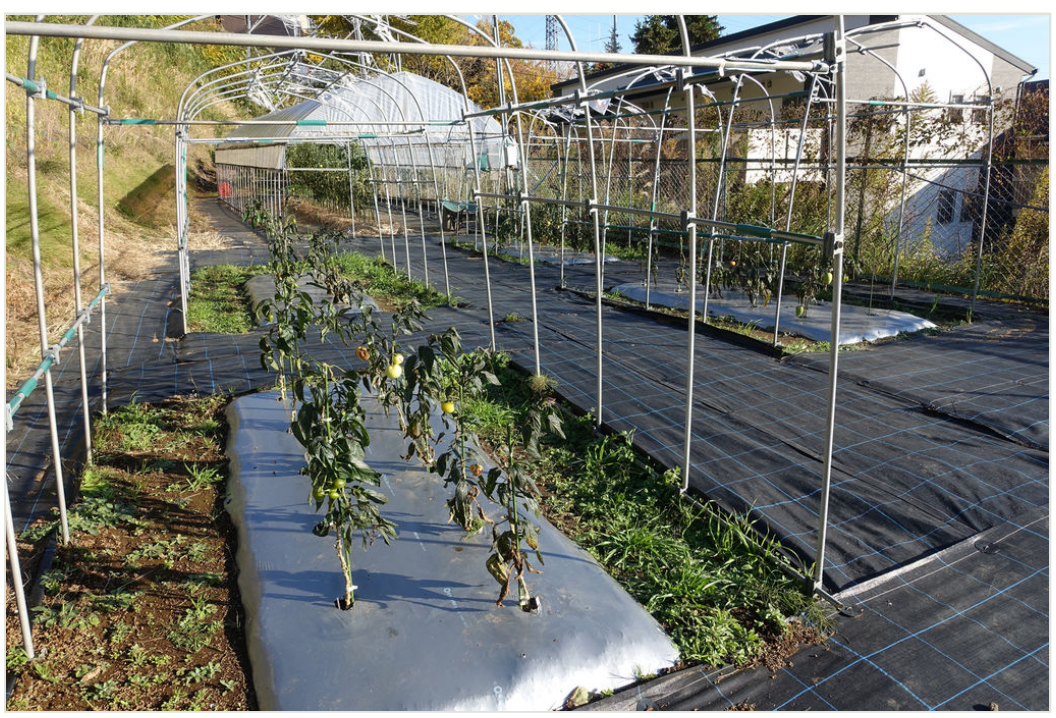

Figure 2. doi

An open field, one of the survey plots in the Atsugi Campus of Tokyo University of Agriculture (TUA), indicated as Plot A in Fig. 1 


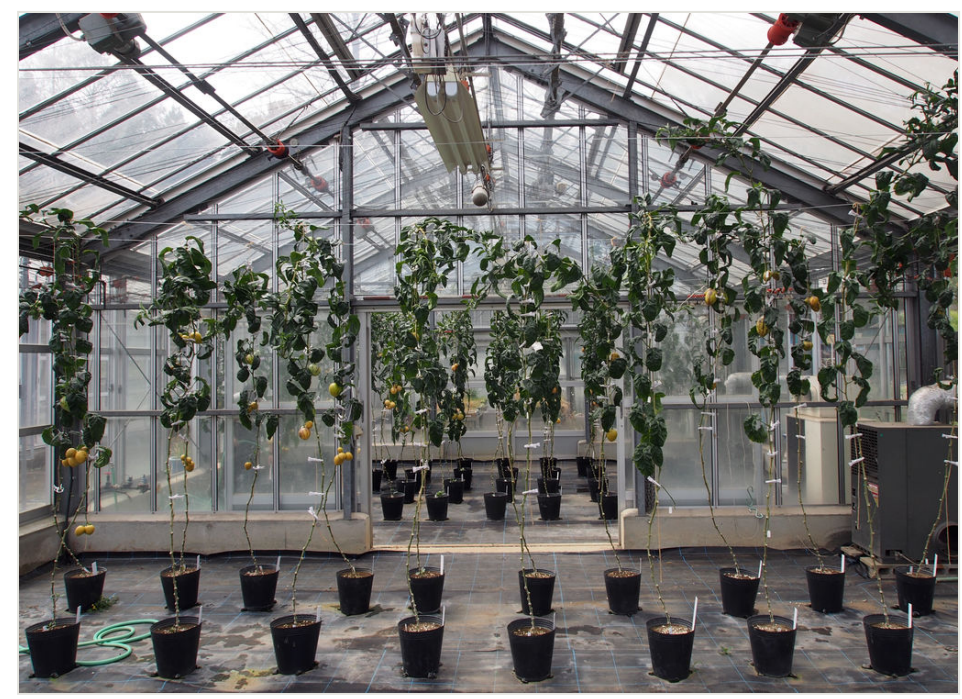

Figure 3. doi

A greenhouse, one of the survey plots in the Atsugi Campus of Tokyo University of Agriculture (TUA), indicated as Plot B in Fig. 1

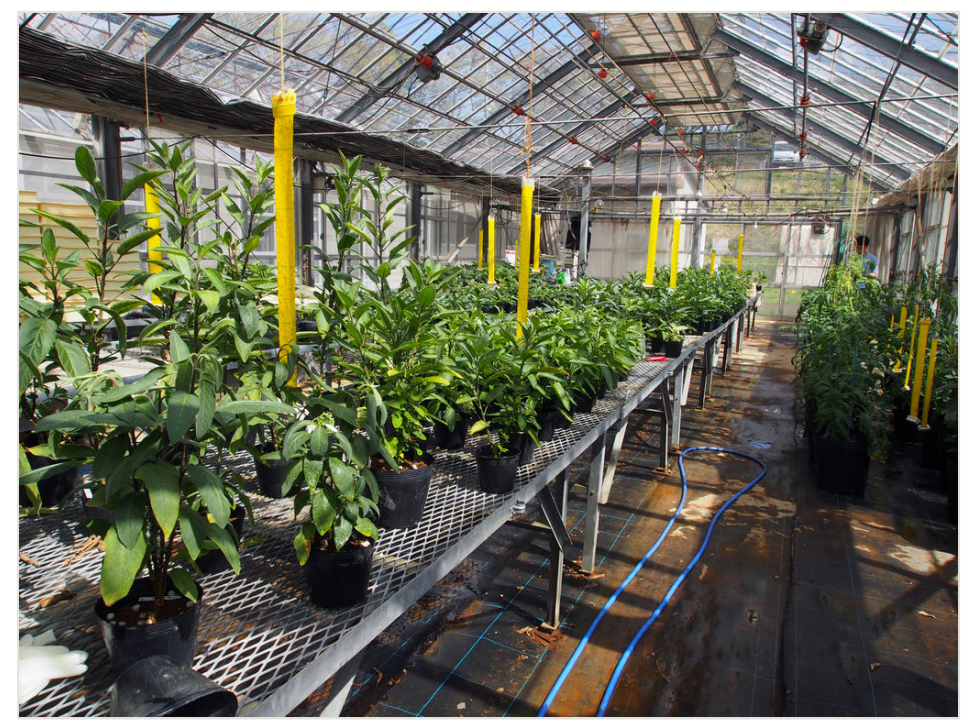

Figure 4. doi

A greenhouse, one of the survey plots in the Atsugi Campus of Tokyo University of Agriculture (TUA), indicated as Plot $\mathrm{C}$ in Fig. 1. 


\section{Sampling methods for insects and mites}

All specimens were collected by beating the leaves and branches of pepino plants after observation in field. A total of 34 collections were performed in the three plots from August 30,2016 to January 21,2017 , for a maximum of $3 \mathrm{~h} /$ day in the daytime. The collected insects were killed immediately after capture, using ethyl acetate; aphids, lepidopteran larvae, and mites were fixed in plastic bottles filled with $70-80 \%$ ethanol. All specimens were prepared as dry mounted, slide mounded, or ethanol preserved for morphological examination.

\section{Identification methods for insects and mites}

Identification of insect and mite specimens was performed using stereoscopic microscopes (Olympus SZ60 and Olympus SZX16, Tokyo, Japan) and optical microscopes (Olympus $\mathrm{BH}-2$ and Olympus BX41, Tokyo, Japan) by $\mathrm{TI}$ and $\mathrm{YY}$ according to the following literature: Ehara and Gotoh (2009), Furukawa (2005), Harada and Takizawa (2012), Iwasaki et al. (2000), Kawai (1980), Masumoto and Okajima (2013), Matsumoto (2008), Moritsu (1983), Tanaka and Uesato (2012), Umeya and Okada (2003), Yasunaga et al. (2001), Yasunaga et al. (2015), along with the original descriptions and/or redescriptions of corresponding species if necessary. Collected specimens were regarded as pests in case these were directly damaging insects or mites on pepino plants, were known as pests of pepino in the native range and introduced regions of pepino other than Japan, or were known as pests of major solanaceous crops such as tomato, eggplant, green pepper, and potato, in Japan, with a reference to The Japanese Society of Applied Entomology and Zoology (2006). All examined specimens are preserved in the Insect Collection (IC) at the Laboratory of Entomology, TUA (LETUA).

\section{Observation of virus-like diseases and virus detection}

We surveyed whether pepino plants showed symptoms of virus infection such as mosaic, mottle, necrosis, or chlorosis. The symptomatic leaves were collected and used for virus detection as follows: Total RNA was extracted from the samples using Trizol reagent (Invitrogen Corp., Carlsbad, CA) according to the manufacturer's instructions. Total RNA was used as a template for first-strand cDNA synthesis by ReverTra Ace $-\alpha-{ }^{\circledR}$ kit (TOYOBO Co., Ltd., Osaka, Japan) followed by DNA amplification using TaKaRa Ex Taq ${ }^{\mathrm{TM}}$ PCR buffer (Takara Bio Inc., Otsu, Japan) with genus-specific or species-specific primers (Table 1). Alfalfa mosaic virus (AMV, genus Alfamovirus) and Cucumber mosaic virus (CMV, genus Cucumovirus) had been reported from pepino plants grown in Kanagawa Prefecture, Japan, additionally, Pepino mosaic virus (PeMV, genus Potexvirus) and two carlaviruses (Potato virus $\mathrm{H}(\mathrm{PVH})$ named tentatively and Potato virus $S$ (PVS) reclassified) had been detected from symptomatic and asymptomatic pepino plants in abroad, respectively. Even though any potyvirus had not been reported from pepino plants so far, we tried here detecting whether it was occurred or not from our samples. Reverse transcriptionpolymerase chain reaction (RT-PCR) products were analyzed by electrophoresis in a $2 \%$ agarose gel. 
Table 1.

Primers used for virus detection in this study.

\begin{tabular}{|c|c|c|c|c|c|}
\hline Target virus & Primer & Strand & Sequence (5' to $\left.3^{\prime}\right)$ & $\begin{array}{l}\text { Expected } \\
\text { amplicon size } \\
\text { (bp) }\end{array}$ & Reference \\
\hline \multirow{2}{*}{$\begin{array}{l}\text { Alfalfa mosaic virus } \\
\text { (AMV) }\end{array}$} & AMV-F2 & + & ATCATGAGTTCTTCACAAAAGAA & \multirow[t]{2}{*}{670} & \multirow{2}{*}{$\begin{array}{l}\text { Xu and Nie } \\
\text { (2006) }\end{array}$} \\
\hline & AMV-R2 & - & TCAATGACGATCAAGATCGTC & & \\
\hline \multirow{2}{*}{$\begin{array}{l}\text { Cucumber mosaic } \\
\text { virus (CMV) }\end{array}$} & CPTALL-5 & + & YASYTTTDRGGTTCAATTCC & \multirow[t]{2}{*}{950} & \multirow[t]{2}{*}{ Choi et al. (1999) } \\
\hline & CPTALL-3 & - & GACTGACCATTTTAGCCG & & \\
\hline \multirow[t]{2}{*}{ Genus Carlavirus } & Carla-uni & + & GGAGTAACCGAGGTGATACC & \multirow[t]{2}{*}{120} & \multirow{2}{*}{$\begin{array}{l}\text { Badge et al. } \\
\text { (1996) modified }\end{array}$} \\
\hline & oligo dT & - & $\mathrm{T}_{18}$ & & \\
\hline \multirow[t]{2}{*}{ Genus Potexvirus } & Potex 5 & + & CAYCARCARGCMAARGAYGA & \multirow[t]{2}{*}{600} & \multirow{2}{*}{$\begin{array}{l}\text { van der Vlugt and } \\
\text { Berendsen } \\
(2002)\end{array}$} \\
\hline & $\begin{array}{l}\text { Potex } \\
2 \mathrm{RC}\end{array}$ & - & AGCATRGCNSCRTCYTG & & \\
\hline \multirow[t]{2}{*}{ Genus Potyvirus } & CIFor & + & GGIVVIGTIGGIWSIGGIAARTCIAC & \multirow[t]{2}{*}{700} & \multirow[t]{2}{*}{ Ha et al. (2008) } \\
\hline & CIRev & - & ACICCRTTYTCDATDATRTTIGTIGC & & \\
\hline
\end{tabular}

\section{Data resources}

In this study, a total of 498 specimens of insects and mites were collected from pepino plants on the three studied plots. Of these specimens, 459 individuals belonging to 11 species were recognized as pests of pepino. They consisted of nine insect species belonging to eight families of five orders and two mite species in two families of one order (Table 2). The remaining specimens (39 individuals) were identified as predatory mites attacking other mites (Acari: Phytoseiidae), parasitic wasps of certain insects (Hymenoptera: Braconidae), and incidental visitors to pepino plants, including grasshoppers (Orthoptera: Tettigoniidae), seed bugs (Hemiptera: Lygaeoidea), and butterflies (Lepidoptera: Papilionidae).

Table 2.

List of insect and mite pests found on pepino plants in the Atsugi Campus of Tokyo University of Agriculture (TUA), Kanagawa, Japan.

\begin{tabular}{|l|l|l|l|l|}
\hline Class & Order & Family & Species & Suvery plots \\
\hline Insecta & Thysanoptera & Thripidae & Frankliniella intonsa (Trybom, 1895) & A C \\
\hline Insecta & Hemiptera & Aleyrodidae & Bemisia tabaci (Gennadius, 1889) & B C \\
\hline
\end{tabular}




\begin{tabular}{|l|l|l|l|l|}
\hline Insecta & Hemiptera & Aphididae & Aphis gossypii Glover, 1877 & A C \\
\hline Insecta & Hemiptera & Pseudococcidae & Phenacoccus solani Ferris, 1918 & C \\
\hline Insecta & Hemiptera & Miridae & Campylomma livida Reuter, 1885 & A \\
\hline Insecta & Coleoptera & Chrysomelidae & Epitrix hirtipennis (Melsheimer, 1847) & A C \\
\hline Insecta & Diptera & Agromyzidae & Liriomyza sativae Blanchard, 1938 & C \\
\hline Insecta & Lepidoptera & Noctuidae & Spodoptera litura (Fabricius, 1775) & A B \\
\hline Insecta & Lepidoptera & Noctuidae & Trichoplusia ni (Hübner, 1803) & B \\
\hline Arachnida & Acari & Tarsonemidae & Polyphagotarsonemus latus (Banks, 1904) & C \\
\hline Arachnida & Acari & Tetranychidae & Tetranychus urticae Koch, 1836 & A C \\
\hline
\end{tabular}

Of these 11 pest species, six were detected from Plot $A$, three from Plot $B$, and eight from Plot C (Table 2). The plant bug species Campylomma livida Reuter, 1885 was found only in the open field (Plot A). Five species, the cotton whitefly Bemisia tabaci (Gennadius, 1889), the mealy bug Phenacoccus solani Ferris, 1918, the tomato leaf miner Liriomyza sativae Blanchard, 1938, the plusiine noctuid caterpillar Trichoplusia ni (Hübner, 1803), and the broad mite Polyphagotarsonemus latus (Banks, 1904), were found only in greenhouses (Plots B and C); the former two are well known as glasshouse pests in Japan. No pest species were common in all the three plots. On an empirical basis, through our survey in the plots, two pest species, the two-spotted spider mite Tetranychus urticae Koch, 1836 and the cotton aphid Aphis gossypii Glover, 1877, were much more abundant on pepino plants than the other pest species recognized, with several hundreds of these two species on each pepino plant.

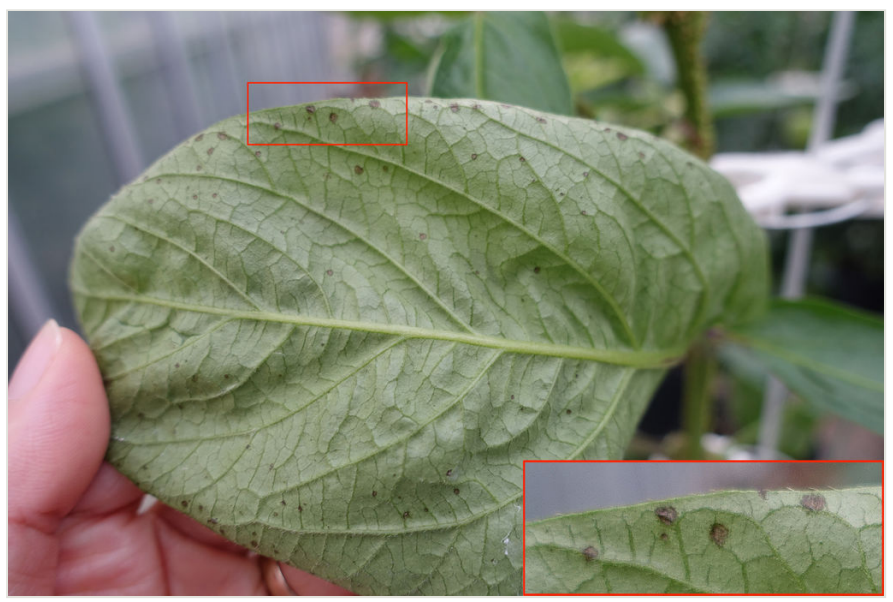

Figure 5. doi

Virus-like symptoms on pepino plants: necrotic spots on pepino leaves in a greenhouse (Fig. 1, Plot C). 
Regarding virus-like diseases in our research fields, pepino leaves showing necrosis were rarely found in the greenhouse (Fig. 5). However, upper leaves showing mottle symptoms with deformation were remarkably observed only in the acaricide-untreated pepinos in the open field (Plot A) during September to October (Fig. 6). Those symptomatic leaves were tested for the detection of Alfalfa mosaic virus (AMV) and Cucumber mosaic virus (CMV), as well as the genera Carlavirus, Potexvirus, and Potyvirus. All tested pepino leaves showed no infection of the above-mentioned viruses (data not shown).

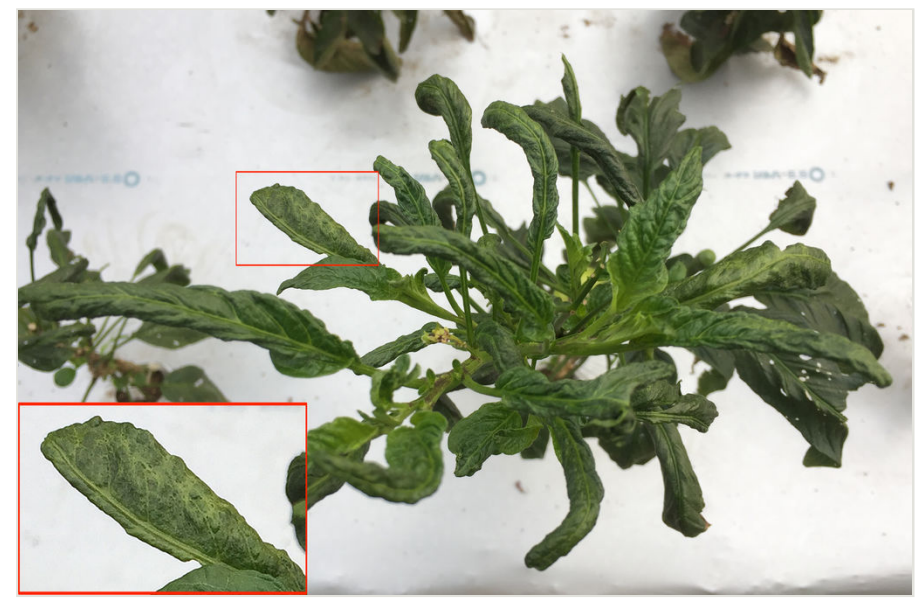

Figure 6. doi

Virus-like symptoms on pepino plants: mottle and deformation on young pepino leaves at an open field (Fig. 1, Plot A).

\section{Checklist of insect and mite pests of pepino in Kanagawa, Japan}

\section{Class Insecta Linnaeus, 1758}

Order Thysanoptera Haliday, 1836

Family Thripidae Stevens, 1829

Frankliniella intonsa (Trybom, 1895)

Materials

a. namePublishedln: 1895; kingdom: Animalia; phylum: Arthropoda; class: Insecta; order: Thysanoptera; family: Thripidae; genus: Frankliniella; specificEpithet: intonsa; scientificNameAuthorship: Trybom; country: Japan; stateProvince: Kanagawa; municipality: Atsugi-shi; locality: Atsugi Campus, Tokyo University of Agriculture, Funako; minimumElevationInMeters: 49; maximumElevationInMeters: 49; decimalLatitude: 
35.431707; decimalLongitude: 139.345165; geodeticDatum: WGS84; samplingProtocol: beating of leaves and branches (including visual searches); eventDate: 2016-09-29; individualCount: 1; lifeStage: adult; recordedBy: Y. Yamada; otherCatalogNumbers: 2017-00005; identifiedBy: Y. Yamada; dateldentified: 2017; institutionCode: LETUA; collectionCode: IC

b. namePublishedln: 1895; kingdom: Animalia; phylum: Arthropoda; class: Insecta; order: Thysanoptera; family: Thripidae; genus: Frankliniella; specificEpithet: intonsa; scientificNameAuthorship: Trybom; country: Japan; stateProvince: Kanagawa; municipality: Atsugi-shi; locality: Atsugi Campus, Tokyo University of Agriculture, Funako; minimumElevationInMeters: 42; maximumElevationInMeters: 42; decimalLatitude: 35.428874; decimalLongitude: 139.34929; geodeticDatum: WGS84; samplingProtocol: beating of leaves and branches (including visual searches); eventDate: 2017-01-07; individualCount: 3; lifeStage: adult; recordedBy: Y. Yamada; otherCatalogNumbers: 2017-00006 | 2017-00007 | 2017-00008; identifiedBy: Y. Yamada; dateldentified: 2017; institutionCode: LETUA; collectionCode: IC

c. namePublishedln: 1895; kingdom: Animalia; phylum: Arthropoda; class: Insecta; order: Thysanoptera; family: Thripidae; genus: Frankliniella; specificEpithet: intonsa; scientificNameAuthorship: Trybom; country: Japan; stateProvince: Kanagawa; municipality: Atsugi-shi; locality: Atsugi Campus, Tokyo University of Agriculture, Funako; minimumElevationInMeters: 42; maximumElevationInMeters: 42; decimalLatitude: 35.428874; decimalLongitude: 139.34929; geodeticDatum: WGS84; samplingProtocol: beating of leaves and branches (including visual searches); eventDate: 2017-01-09; individualCount: 1; lifeStage: adult; recordedBy: Y. Yamada; otherCatalogNumbers: 2017-00009; identifiedBy: Y. Yamada; dateldentified: 2017; institutionCode: LETUA; collectionCode: IC

\section{Order Hemiptera Linnaeus, 1758}

\section{Family Aleyrodidae Westwood, 1840}

\section{Bemisia tabaci (Gennadius, 1889)}

\section{Materials}

a. namePublishedln: 1889; kingdom: Animalia; phylum: Arthropoda; class: Insecta; order: Hemiptera; family: Aleyrodidae; genus: Bemisia; specificEpithet: tabaci; scientificNameAuthorship: Gennadius; country: Japan; stateProvince: Kanagawa; municipality: Atsugi-shi; locality: Atsugi Campus, Tokyo University of Agriculture, Funako; minimumElevationInMeters: 27; maximumElevationInMeters: 27; decimalLatitude: 35.43043; decimalLongitude: 139.349516; geodeticDatum: WGS84; samplingProtocol: beating of leaves and branches (including visual searches); eventDate: 2016-12-18; individualCount: 1; lifeStage: adult; recordedBy: K. Takahata; otherCatalogNumbers: 2017-00010; identifiedBy: Y. Yamada; dateldentified: 2017; institutionCode: LETUA; collectionCode: IC

b. namePublishedln: 1889; kingdom: Animalia; phylum: Arthropoda; class: Insecta; order: Hemiptera; family: Aleyrodidae; genus: Bemisia; specificEpithet: tabaci; scientificNameAuthorship: Gennadius; country: Japan; stateProvince: Kanagawa; municipality: Atsugi-shi; locality: Atsugi Campus, Tokyo University of Agriculture, Funako; minimumElevationInMeters: 42; maximumElevationInMeters: 42; decimalLatitude: 
35.428874; decimalLongitude: 139.34929; geodeticDatum: WGS84; samplingProtocol: beating of leaves and branches (including visual searches); eventDate: 2016-12-19; individualCount: 3; lifeStage: adult; recordedBy: Y. Yamada; otherCatalogNumbers: 2017-00011 | 2017-00012 | 2017-00013; identifiedBy: Y. Yamada; dateldentified: 2017; institutionCode: LETUA; collectionCode: IC

c. namePublishedln: 1889; kingdom: Animalia; phylum: Arthropoda; class: Insecta; order: Hemiptera; family: Aleyrodidae; genus: Bemisia; specificEpithet: tabaci; scientificNameAuthorship: Gennadius; country: Japan; stateProvince: Kanagawa; municipality: Atsugi-shi; locality: Atsugi Campus, Tokyo University of Agriculture, Funako; minimumElevationInMeters: 42; maximumElevationInMeters: 42; decimalLatitude: 35.428874; decimalLongitude: 139.34929; geodeticDatum: WGS84; samplingProtocol: beating of leaves and branches (including visual searches); eventDate: 2017-01-09; individualCount: 2; lifeStage: adult; recordedBy: Y. Yamada; otherCatalogNumbers: 2017-00014 | 2017-00015; identifiedBy: Y. Yamada; dateldentified: 2017; institutionCode: LETUA; collectionCode: IC

\section{Family Aphididae Latreille, 1802}

\section{Aphis gossypii Glover, 1877}

\section{Materials}

a. namePublishedln: 1877; kingdom: Animalia; phylum: Arthropoda; class: Insecta; order: Hemiptera; family: Aphididae; genus: Aphis; specificEpithet: gossypii; scientificNameAuthorship: Glover; country: Japan; stateProvince: Kanagawa; municipality: Atsugi-shi; locality: Atsugi Campus, Tokyo University of Agriculture, Funako; minimumElevationInMeters: 49; maximumElevationInMeters: 49; decimalLatitude: 35.431707; decimalLongitude: 139.345165; geodeticDatum: WGS84; samplingProtocol: beating of leaves and branches (including visual searches); eventDate: 2016-08-30; individualCount: 3; lifeStage: adult; recordedBy: T. Ishikawa; otherCatalogNumbers: 2017-00016 | 2017-00017 | 2017-00018; identifiedBy: Y. Yamada; dateldentified: 2017; institutionCode: LETUA; collectionCode: IC

b. namePublishedln: 1877; kingdom: Animalia; phylum: Arthropoda; class: Insecta; order: Hemiptera; family: Aphididae; genus: Aphis; specificEpithet: gossypii; scientificNameAuthorship: Glover; country: Japan; stateProvince: Kanagawa; municipality: Atsugi-shi; locality: Atsugi Campus, Tokyo University of Agriculture, Funako; minimumElevationInMeters: 49; maximumElevationInMeters: 49; decimalLatitude: 35.431707; decimalLongitude: 139.345165; geodeticDatum: WGS84; samplingProtocol: beating of leaves and branches (including visual searches); eventDate: 2016-09-28; individualCount: 3; lifeStage: adult; recordedBy: Y. Yamada; otherCatalogNumbers: 2017-00019 | 2017-00020 | 2017-00021; identifiedBy: Y. Yamada; dateldentified: 2017; institutionCode: LETUA; collectionCode: IC

c. namePublishedln: 1877; kingdom: Animalia; phylum: Arthropoda; class: Insecta; order: Hemiptera; family: Aphididae; genus: Aphis; specificEpithet: gossypii; scientificNameAuthorship: Glover; country: Japan; stateProvince: Kanagawa; municipality: Atsugi-shi; locality: Atsugi Campus, Tokyo University of Agriculture, Funako; minimumElevationInMeters: 49; maximumElevationInMeters: 49; decimalLatitude: 35.431707; decimalLongitude: 139.345165; geodeticDatum: WGS84; samplingProtocol: beating of leaves and branches (including visual searches); eventDate: 2016-10-06; individualCount: 1; lifeStage: adult; recordedBy: Y. Yamada; otherCatalogNumbers: 
2017-00022; identifiedBy: Y. Yamada; dateldentified: 2017; institutionCode: LETUA; collectionCode: IC

d. namePublishedln: 1877; kingdom: Animalia; phylum: Arthropoda; class: Insecta; order: Hemiptera; family: Aphididae; genus: Aphis; specificEpithet: gossypii; scientificNameAuthorship: Glover; country: Japan; stateProvince: Kanagawa; municipality: Atsugi-shi; locality: Atsugi Campus, Tokyo University of Agriculture, Funako; minimumElevationInMeters: 49; maximumElevationInMeters: 49; decimalLatitude: 35.431707; decimalLongitude: 139.345165; geodeticDatum: WGS84; samplingProtocol: beating of leaves and branches (including visual searches); eventDate: 2016-11-08; individualCount: 1; lifeStage: adult; recordedBy: Y. Yamada; otherCatalogNumbers: 2017-00023; identifiedBy: Y. Yamada; dateldentified: 2017; institutionCode: LETUA; collectionCode: IC

e. namePublishedln: 1877; kingdom: Animalia; phylum: Arthropoda; class: Insecta; order: Hemiptera; family: Aphididae; genus: Aphis; specificEpithet: gossypii; scientificNameAuthorship: Glover; country: Japan; stateProvince: Kanagawa; municipality: Atsugi-shi; locality: Atsugi Campus, Tokyo University of Agriculture, Funako; minimumElevationInMeters: 49; maximumElevationInMeters: 49; decimalLatitude: 35.431707; decimalLongitude: 139.345165; geodeticDatum: WGS84; samplingProtocol: beating of leaves and branches (including visual searches); eventDate: 2016-11-10; individualCount: 1; lifeStage: adult; recordedBy: Y. Yamada; otherCatalogNumbers: 2017-00024; identifiedBy: Y. Yamada; dateldentified: 2017; institutionCode: LETUA; collectionCode: IC

f. namePublishedln: 1877; kingdom: Animalia; phylum: Arthropoda; class: Insecta; order: Hemiptera; family: Aphididae; genus: Aphis; specificEpithet: gossypii; scientificNameAuthorship: Glover; country: Japan; stateProvince: Kanagawa; municipality: Atsugi-shi; locality: Atsugi Campus, Tokyo University of Agriculture, Funako; minimumElevationInMeters: 49; maximumElevationInMeters: 49; decimalLatitude: 35.431707; decimalLongitude: 139.345165; geodeticDatum: WGS84; samplingProtocol: beating of leaves and branches (including visual searches); eventDate: 2016-11-17; individualCount: 1; lifeStage: adult; recordedBy: Y. Yamada; otherCatalogNumbers: 2017-00025; identifiedBy: Y. Yamada; dateldentified: 2017; institutionCode: LETUA; collectionCode: IC

g. namePublishedln: 1877; kingdom: Animalia; phylum: Arthropoda; class: Insecta; order: Hemiptera; family: Aphididae; genus: Aphis; specificEpithet: gossypii; scientificNameAuthorship: Glover; country: Japan; stateProvince: Kanagawa; municipality: Atsugi-shi; locality: Atsugi Campus, Tokyo University of Agriculture, Funako; minimumElevationInMeters: 49; maximumElevationInMeters: 49; decimalLatitude: 35.431707; decimalLongitude: 139.345165; geodeticDatum: WGS84; samplingProtocol: beating of leaves and branches (including visual searches); eventDate: 2016-11-30; individualCount: 4; lifeStage: adult; recordedBy: Y. Yamada; otherCatalogNumbers: 2017-00026 | 2017-00027 | 2017-00028 | 2017-00029; identifiedBy: Y. Yamada; dateldentified: 2017; institutionCode: LETUA; collectionCode: IC

h. namePublishedln: 1877; kingdom: Animalia; phylum: Arthropoda; class: Insecta; order: Hemiptera; family: Aphididae; genus: Aphis; specificEpithet: gossypii; scientificNameAuthorship: Glover; country: Japan; stateProvince: Kanagawa; municipality: Atsugi-shi; locality: Atsugi Campus, Tokyo University of Agriculture, Funako; minimumElevationInMeters: 42; maximumElevationInMeters: 42; decimalLatitude: 35.428874; decimalLongitude: 139.34929; geodeticDatum: WGS84; samplingProtocol: beating of leaves and branches (including visual searches); eventDate: 2016-12-26; individualCount: 17; lifeStage: adult; recordedBy: Y. Yamada; otherCatalogNumbers: 
| 2017-00036 | 2017-00037 | 2017-00038 | 2017-00039 | 2017-00040 | 2017-00041 | 2017-00042 | 2017-00043 | 2017-00044 | 2017-00047 | 2017-00048; identifiedBy: Y. Yamada; dateldentified: 2017; institutionCode: LETUA; collectionCode: IC

i. namePublishedln: 1877; kingdom: Animalia; phylum: Arthropoda; class: Insecta; order: Hemiptera; family: Aphididae; genus: Aphis; specificEpithet: gossypii; scientificNameAuthorship: Glover; country: Japan; stateProvince: Kanagawa; municipality: Atsugi-shi; locality: Atsugi Campus, Tokyo University of Agriculture, Funako; minimumElevationInMeters: 42; maximumElevationInMeters: 42; decimalLatitude: 35.428874; decimalLongitude: 139.34929; geodeticDatum: WGS84; samplingProtocol: beating of leaves and branches (including visual searches); eventDate: 2016-12-26; individualCount: 2; lifeStage: nymph; recordedBy: Y. Yamada; otherCatalogNumbers: 2017-00045 | 2017-00046; identifiedBy: Y. Yamada; dateldentified: 2017; institutionCode: LETUA; collectionCode: IC

j. namePublishedln: 1877; kingdom: Animalia; phylum: Arthropoda; class: Insecta; order: Hemiptera; family: Aphididae; genus: Aphis; specificEpithet: gossypii; scientificNameAuthorship: Glover; country: Japan; stateProvince: Kanagawa; municipality: Atsugi-shi; locality: Atsugi Campus, Tokyo University of Agriculture, Funako; minimumElevationInMeters: 42; maximumElevationInMeters: 42; decimalLatitude: 35.428874; decimalLongitude: 139.34929; geodeticDatum: WGS84; samplingProtocol: beating of leaves and branches (including visual searches); eventDate: 2016-12-30; individualCount: 30; lifeStage: adult; recordedBy: Y. Yamada; otherCatalogNumbers: 2017-00049 | 2017-00050 | 2017-00051 | 2017-00052 | 2017-00053 | 2017-00054 | | | | 2017-00073 | 2017-00074 | 2017-00075 | 2017-00078 | 2017-00079 | 2017-00080; identifiedBy: Y. Yamada; dateldentified: 2017; institutionCode: LETUA; collectionCode: IC k. namePublishedln: 1877; kingdom: Animalia; phylum: Arthropoda; class: Insecta; order: Hemiptera; family: Aphididae; genus: Aphis; specificEpithet: gossypii; scientificNameAuthorship: Glover; country: Japan; stateProvince: Kanagawa; municipality: Atsugi-shi; locality: Atsugi Campus, Tokyo University of Agriculture, Funako; minimumElevationInMeters: 42; maximumElevationInMeters: 42; decimalLatitude: 35.428874; decimalLongitude: 139.34929; geodeticDatum: WGS84; samplingProtocol: beating of leaves and branches (including visual searches); eventDate: 2016-12-30; individualCount: 2; lifeStage: nymph; recordedBy: Y. Yamada; otherCatalogNumbers: 2017-00076 | 2017-00077; identifiedBy: Y. Yamada; dateldentified: 2017; institutionCode: LETUA; collectionCode: IC

I. namePublishedln: 1877; kingdom: Animalia; phylum: Arthropoda; class: Insecta; order: Hemiptera; family: Aphididae; genus: Aphis; specificEpithet: gossypii; scientificNameAuthorship: Glover; country: Japan; stateProvince: Kanagawa; municipality: Atsugi-shi; locality: Atsugi Campus, Tokyo University of Agriculture, Funako; minimumElevationInMeters: 42; maximumElevationInMeters: 42; decimalLatitude: 35.428874; decimalLongitude: 139.34929; geodeticDatum: WGS84; samplingProtocol: beating of leaves and branches (including visual searches); eventDate: 2017-01-03; individualCount: 48; lifeStage: adult; recordedBy: Y. Yamada; otherCatalogNumbers: | 2017-00081 | 2017-00082 | 2017-00083 | 2017-00084 | 2017-00085 | 2017-00086 | | | | 
| 2017-00111 | 2017-00112 | 2017-00113 | 2017-00114 | 2017-00115 | 2017-00116 | 2017-00117 | 2017-00118 | 2017-00119 | 2017-00120 | 2017-00121 | 2017-00122 | 2017-00131 | 2017-00132 | 2017-00133 | 2017-00134 | 2017-00135 | 2017-00136; identifiedBy: Y. Yamada; dateldentified: 2017; institutionCode: LETUA; collectionCode: IC

m. namePublishedln: 1877; kingdom: Animalia; phylum: Arthropoda; class: Insecta; order: Hemiptera; family: Aphididae; genus: Aphis; specificEpithet: gossypii; scientificNameAuthorship: Glover; country: Japan; stateProvince: Kanagawa; municipality: Atsugi-shi; locality: Atsugi Campus, Tokyo University of Agriculture, Funako; minimumElevationInMeters: 42; maximumElevationInMeters: 42; decimalLatitude: 35.428874; decimalLongitude: 139.34929; geodeticDatum: WGS84; samplingProtocol: beating of leaves and branches (including visual searches); eventDate: 2017-01-03; individualCount: 8; lifeStage: nymph; recordedBy: Y. Yamada; otherCatalogNumbers: | 2017-00129 | 2017-00130; identifiedBy: Y. Yamada; dateldentified: 2017; institutionCode: LETUA; collectionCode: IC

n. namePublishedln: 1877; kingdom: Animalia; phylum: Arthropoda; class: Insecta; order: Hemiptera; family: Aphididae; genus: Aphis; specificEpithet: gossypii; scientificNameAuthorship: Glover; country: Japan; stateProvince: Kanagawa; municipality: Atsugi-shi; locality: Atsugi Campus, Tokyo University of Agriculture, Funako; minimumElevationInMeters: 42; maximumElevationInMeters: 42; decimalLatitude: 35.428874; decimalLongitude: 139.34929; geodeticDatum: WGS84; samplingProtocol: beating of leaves and branches (including visual searches); eventDate: 2017-01-05; individualCount: 36; lifeStage: adult; recordedBy: Y. Yamada; otherCatalogNumbers: 2017-00137 | 2017-00138 | 2017-00139 | 2017-00140 | 2017-00141 | 2017-00142 | | | | 2017-00161 | 2017-00162 | 2017-00163 | 2017-00164 | 2017-00165 | 2017-00166 | 2017-00167 | 2017-00168 | 2017-00169 | 2017-00170 | 2017-00171 | 2017-00172; identifiedBy: Y. Yamada; dateldentified: 2017; institutionCode: LETUA; collectionCode: IC o. namePublishedln: 1877; kingdom: Animalia; phylum: Arthropoda; class: Insecta; order: Hemiptera; family: Aphididae; genus: Aphis; specificEpithet: gossypii; scientificNameAuthorship: Glover; country: Japan; stateProvince: Kanagawa; municipality: Atsugi-shi; locality: Atsugi Campus, Tokyo University of Agriculture, Funako; minimumElevationInMeters: 42; maximumElevationInMeters: 42; decimalLatitude: 35.428874; decimalLongitude: 139.34929; geodeticDatum: WGS84; samplingProtocol: beating of leaves and branches (including visual searches); eventDate: 2017-01-07; individualCount: 25; lifeStage: adult; recordedBy: Y. Yamada; otherCatalogNumbers: | | | | 2017-00197; identifiedBy: Y. Yamada; dateldentified: 2017; institutionCode: LETUA; collectionCode: IC

p. namePublishedln: 1877; kingdom: Animalia; phylum: Arthropoda; class: Insecta; order: Hemiptera; family: Aphididae; genus: Aphis; specificEpithet: gossypii; scientificNameAuthorship: Glover; country: Japan; stateProvince: Kanagawa; municipality: Atsugi-shi; locality: Atsugi Campus, Tokyo University of Agriculture, Funako; minimumElevationInMeters: 42; maximumElevationInMeters: 42; decimalLatitude: 
35.428874; decimalLongitude: 139.34929; geodeticDatum: WGS84; samplingProtocol: beating of leaves and branches (including visual searches); eventDate: 2017-01-09; individualCount: 4; lifeStage: adult; recordedBy: Y. Yamada; otherCatalogNumbers: 2017-00198 | 2017-00199 | 2017-00200 | 2017-00201; identifiedBy: Y. Yamada; dateldentified: 2017; institutionCode: LETUA; collectionCode: IC

q. namePublishedln: 1877; kingdom: Animalia; phylum: Arthropoda; class: Insecta; order: Hemiptera; family: Aphididae; genus: Aphis; specificEpithet: gossypii; scientificNameAuthorship: Glover; country: Japan; stateProvince: Kanagawa; municipality: Atsugi-shi; locality: Atsugi Campus, Tokyo University of Agriculture, Funako; minimumElevationInMeters: 42; maximumElevationInMeters: 42; decimalLatitude: 35.428874; decimalLongitude: 139.34929; geodeticDatum: WGS84; samplingProtocol: beating of leaves and branches (including visual searches); eventDate: 2017-01-12; individualCount: 43; lifeStage: adult; recordedBy: Y. Yamada; otherCatalogNumbers: | | | | | | 2017-00238 | 2017-00239 | 2017-00240 | 2017-00241 | 2017-00242 | 2017-00243 | 2017-00244; identifiedBy: Y. Yamada; dateldentified: 2017; institutionCode: LETUA; collectionCode: IC

r. namePublishedIn: 1877; kingdom: Animalia; phylum: Arthropoda; class: Insecta; order: Hemiptera; family: Aphididae; genus: Aphis; specificEpithet: gossypii; scientificNameAuthorship: Glover; country: Japan; stateProvince: Kanagawa; municipality: Atsugi-shi; locality: Atsugi Campus, Tokyo University of Agriculture, Funako; minimumElevationInMeters: 42; maximumElevationInMeters: 42; decimalLatitude: 35.428874; decimalLongitude: 139.34929; geodeticDatum: WGS84; samplingProtocol: beating of leaves and branches (including visual searches); eventDate: 2017-01-16; individualCount: 22; lifeStage: adult; recordedBy: Y. Yamada; otherCatalogNumbers: 2017-00245 | 2017-00246 | 2017-00247 | 2017-00248 | 2017-00249 | 2017-00250 | | | 2017-00263 | 2017-00264 | 2017-00265 | 2017-00266; identifiedBy: Y. Yamada; dateldentified: 2017; institutionCode: LETUA; collectionCode: IC

s. namePublishedln: 1877; kingdom: Animalia; phylum: Arthropoda; class: Insecta; order: Hemiptera; family: Aphididae; genus: Aphis; specificEpithet: gossypii; scientificNameAuthorship: Glover; country: Japan; stateProvince: Kanagawa; municipality: Atsugi-shi; locality: Atsugi Campus, Tokyo University of Agriculture, Funako; minimumElevationInMeters: 42; maximumElevationInMeters: 42; decimalLatitude: 35.428874; decimalLongitude: 139.34929; geodeticDatum: WGS84; samplingProtocol: beating of leaves and branches (including visual searches); eventDate: 2017-01-21; individualCount: 3; lifeStage: adult; recordedBy: Y. Yamada; otherCatalogNumbers: 2017-00267 | 2017-00268 | 2017-00269; identifiedBy: Y. Yamada; dateldentified: 2017; institutionCode: LETUA; collectionCode: IC 


\section{Family Pseudococcidae Heymons, 1915}

\section{Phenacoccus solani Ferris, 1918}

\section{Materials}

a. namePublishedln: 1918; kingdom: Animalia; phylum: Arthropoda; class: Insecta; order: Hemiptera; family: Pseudococcidae; genus: Phenacoccus; specificEpithet: solani; scientificNameAuthorship: Ferris; country: Japan; stateProvince: Kanagawa; municipality: Atsugi-shi; locality: Atsugi Campus, Tokyo University of Agriculture, Funako; minimumElevationInMeters: 42; maximumElevationInMeters: 42; decimalLatitude: 35.428874; decimalLongitude: 139.34929; geodeticDatum: WGS84; samplingProtocol: beating of leaves and branches (including visual searches); eventDate: 2016-12-30; individualCount: 1; lifeStage: nymph; recordedBy: Y. Yamada; otherCatalogNumbers: 2017-00270; identifiedBy: Y. Yamada; dateldentified: 2017; institutionCode: LETUA; collectionCode: IC

b. namePublishedln: 1918; kingdom: Animalia; phylum: Arthropoda; class: Insecta; order: Hemiptera; family: Pseudococcidae; genus: Phenacoccus; specificEpithet: solani; scientificNameAuthorship: Ferris; country: Japan; stateProvince: Kanagawa; municipality: Atsugi-shi; locality: Atsugi Campus, Tokyo University of Agriculture, Funako; minimumElevationInMeters: 42; maximumElevationInMeters: 42; decimalLatitude: 35.428874; decimalLongitude: 139.34929; geodeticDatum: WGS84; samplingProtocol: beating of leaves and branches (including visual searches); eventDate: 2017-01-15; individualCount: 2; lifeStage: adult; recordedBy: Y. Yamada; otherCatalogNumbers: 2017-00271 | 2017-00272; identifiedBy: Y. Yamada; dateldentified: 2017; institutionCode: LETUA; collectionCode: IC

\section{Family Miridae Hahn, 1833}

\section{Campylomma livida Reuter, 1885}

\section{Materials}

a. namePublishedln: 1885; kingdom: Animalia; phylum: Arthropoda; class: Insecta; order: Hemiptera; family: Miridae; genus: Campylomma; specificEpithet: livida; scientificNameAuthorship: Reuter; country: Japan; stateProvince: Kanagawa; municipality: Atsugi-shi; locality: Atsugi Campus, Tokyo University of Agriculture, Funako; minimumElevationInMeters: 49; maximumElevationInMeters: 49; decimalLatitude: 35.431707; decimalLongitude: 139.345165; geodeticDatum: WGS84; samplingProtocol: beating of leaves and branches (including visual searches); eventDate: 2016-11-10; individualCount: 1; lifeStage: nymph; recordedBy: Y. Yamada; otherCatalogNumbers: 2017-00273; identifiedBy: Y. Yamada; dateldentified: 2017; institutionCode: LETUA; collectionCode: IC

b. namePublishedln: 1885; kingdom: Animalia; phylum: Arthropoda; class: Insecta; order: Hemiptera; family: Miridae; genus: Campylomma; specificEpithet: livida; scientificNameAuthorship: Reuter; country: Japan; stateProvince: Kanagawa; municipality: Atsugi-shi; locality: Atsugi Campus, Tokyo University of Agriculture, Funako; minimumElevationInMeters: 49; maximumElevationInMeters: 49; decimalLatitude: 35.431707; decimalLongitude: 139.345165; geodeticDatum: WGS84; samplingProtocol: 
beating of leaves and branches (including visual searches); eventDate: 2016-11-10; individualCount: 1; lifeStage: adult; recordedBy: Y. Yamada; otherCatalogNumbers: 2017-00274; identifiedBy: Y. Yamada; dateldentified: 2017; institutionCode: LETUA; collectionCode: IC

c. namePublishedln: 1885; kingdom: Animalia; phylum: Arthropoda; class: Insecta; order: Hemiptera; family: Miridae; genus: Campylomma; specificEpithet: livida; scientificNameAuthorship: Reuter; country: Japan; stateProvince: Kanagawa; municipality: Atsugi-shi; locality: Atsugi Campus, Tokyo University of Agriculture, Funako; minimumElevationInMeters: 49; maximumElevationInMeters: 49; decimalLatitude: 35.431707; decimalLongitude: 139.345165; geodeticDatum: WGS84; samplingProtocol: beating of leaves and branches (including visual searches); eventDate: 2016-11-15; individualCount: 1; lifeStage: adult; recordedBy: Y. Yamada; otherCatalogNumbers: 2017-00275; identifiedBy: Y. Yamada; dateldentified: 2017; institutionCode: LETUA; collectionCode: IC

d. namePublishedln: 1885; kingdom: Animalia; phylum: Arthropoda; class: Insecta; order: Hemiptera; family: Miridae; genus: Campylomma; specificEpithet: livida; scientificNameAuthorship: Reuter; country: Japan; stateProvince: Kanagawa; municipality: Atsugi-shi; locality: Atsugi Campus, Tokyo University of Agriculture, Funako; minimumElevationInMeters: 49; maximumElevationInMeters: 49; decimalLatitude: 35.431707; decimalLongitude: 139.345165; geodeticDatum: WGS84; samplingProtocol: beating of leaves and branches (including visual searches); eventDate: 2016-11-17; individualCount: 1; lifeStage: adult; recordedBy: Y. Yamada; otherCatalogNumbers: 2017-00276; identifiedBy: Y. Yamada; dateldentified: 2017; institutionCode: LETUA; collectionCode: IC

e. $\quad$ namePublishedln: 1885; kingdom: Animalia; phylum: Arthropoda; class: Insecta; order: Hemiptera; family: Miridae; genus: Campylomma; specificEpithet: livida; scientificNameAuthorship: Reuter; country: Japan; stateProvince: Kanagawa; municipality: Atsugi-shi; locality: Atsugi Campus, Tokyo University of Agriculture, Funako; minimumElevationInMeters: 49; maximumElevationInMeters: 49; decimalLatitude: 35.431707; decimalLongitude: 139.345165; geodeticDatum: WGS84; samplingProtocol: beating of leaves and branches (including visual searches); eventDate: 2016-11-17; individualCount: 1; lifeStage: nymph; recordedBy: Y. Yamada; otherCatalogNumbers: 2017-00277; identifiedBy: Y. Yamada; dateldentified: 2017; institutionCode: LETUA; collectionCode: IC

f. namePublishedln: 1885; kingdom: Animalia; phylum: Arthropoda; class: Insecta; order: Hemiptera; family: Miridae; genus: Campylomma; specificEpithet: livida; scientificNameAuthorship: Reuter; country: Japan; stateProvince: Kanagawa; municipality: Atsugi-shi; locality: Atsugi Campus, Tokyo University of Agriculture, Funako; minimumElevationInMeters: 49; maximumElevationInMeters: 49; decimalLatitude: 35.431707; decimalLongitude: 139.345165; geodeticDatum: WGS84; samplingProtocol: beating of leaves and branches (including visual searches); eventDate: 2016-11-23; individualCount: 1; lifeStage: nymph; recordedBy: Y. Yamada; otherCatalogNumbers: 2017-00278; identifiedBy: Y. Yamada; dateldentified: 2017; institutionCode: LETUA; collectionCode: IC

g. namePublishedln: 1885; kingdom: Animalia; phylum: Arthropoda; class: Insecta; order: Hemiptera; family: Miridae; genus: Campylomma; specificEpithet: livida; scientificNameAuthorship: Reuter; country: Japan; stateProvince: Kanagawa; municipality: Atsugi-shi; locality: Atsugi Campus, Tokyo University of Agriculture, Funako; minimumElevationInMeters: 49; maximumElevationInMeters: 49; decimalLatitude: 35.431707; decimalLongitude: 139.345165; geodeticDatum: WGS84; samplingProtocol: 
beating of leaves and branches (including visual searches); eventDate: 2016-11-23; individualCount: 1; lifeStage: adult; recordedBy: Y. Yamada; otherCatalogNumbers: 2017-00279; identifiedBy: Y. Yamada; dateldentified: 2017; institutionCode: LETUA; collectionCode: IC

h. namePublishedln: 1885; kingdom: Animalia; phylum: Arthropoda; class: Insecta; order: Hemiptera; family: Miridae; genus: Campylomma; specificEpithet: livida; scientificNameAuthorship: Reuter; country: Japan; stateProvince: Kanagawa; municipality: Atsugi-shi; locality: Atsugi Campus, Tokyo University of Agriculture, Funako; minimumElevationInMeters: 49; maximumElevationInMeters: 49; decimalLatitude: 35.431707; decimalLongitude: 139.345165; geodeticDatum: WGS84; samplingProtocol: beating of leaves and branches (including visual searches); eventDate: 2016-11-25; individualCount: 1; lifeStage: adult; recordedBy: Y. Yamada; otherCatalogNumbers: 2017-00280; identifiedBy: Y. Yamada; dateldentified: 2017; institutionCode: LETUA; collectionCode: IC

i. namePublishedln: 1885; kingdom: Animalia; phylum: Arthropoda; class: Insecta; order: Hemiptera; family: Miridae; genus: Campylomma; specificEpithet: livida; scientificNameAuthorship: Reuter; country: Japan; stateProvince: Kanagawa; municipality: Atsugi-shi; locality: Atsugi Campus, Tokyo University of Agriculture, Funako; minimumElevationInMeters: 49; maximumElevationInMeters: 49; decimalLatitude: 35.431707; decimalLongitude: 139.345165; geodeticDatum: WGS84; samplingProtocol: beating of leaves and branches (including visual searches); eventDate: 2016-11-30; individualCount: 1; lifeStage: adult; recordedBy: Y. Yamada; otherCatalogNumbers: 2017-00281; identifiedBy: Y. Yamada; dateldentified: 2017; institutionCode: LETUA; collectionCode: IC

j. namePublishedln: 1885; kingdom: Animalia; phylum: Arthropoda; class: Insecta; order: Hemiptera; family: Miridae; genus: Campylomma; specificEpithet: livida; scientificNameAuthorship: Reuter; country: Japan; stateProvince: Kanagawa; municipality: Atsugi-shi; locality: Atsugi Campus, Tokyo University of Agriculture, Funako; minimumElevationInMeters: 49; maximumElevationInMeters: 49; decimalLatitude: 35.431707; decimalLongitude: 139.345165; geodeticDatum: WGS84; samplingProtocol: beating of leaves and branches (including visual searches); eventDate: 2016-11-30; individualCount: 2; lifeStage: nymph; recordedBy: Y. Yamada; otherCatalogNumbers: 2017-00282 | 2017-00283; identifiedBy: Y. Yamada; dateldentified: 2017; institutionCode: LETUA; collectionCode: IC

\section{Order Coleoptera Linnaeus, 1758}

\section{Family Chrysomelidae Latreille, 1802}

\section{Epitrix hirtipennis (Melsheimer, 1847)}

\section{Materials}

a. namePublishedln: 1847; kingdom: Animalia; phylum: Arthropoda; class: Insecta; order: Coleoptera; family: Chrysomelidae; genus: Epitrix; specificEpithet: hirtipennis; scientificNameAuthorship: Melsheimer; country: Japan; stateProvince: Kanagawa; municipality: Atsugi-shi; locality: Atsugi Campus, Tokyo University of Agriculture, Funako; minimumElevationInMeters: 49; maximumElevationInMeters: 49; decimalLatitude: 35.431707; decimalLongitude: 139.345165; geodeticDatum: WGS84; samplingProtocol: 
beating of leaves and branches (including visual searches); eventDate: 2016-08-30; individualCount: 12; lifeStage: adult; recordedBy: T. Ishikawa; otherCatalogNumbers: 2017-00284 | 2017-00285 | 2017-00286 | 2017-00287 | 2017-00288 | 2017-00289 | 2017-00290 | 2017-00291 | 2017-00292 | 2017-00293 | 2017-00294 | 2017-00295; identifiedBy: Y. Yamada; dateldentified: 2017; institutionCode: LETUA; collectionCode: IC

b. namePublishedln: 1847; kingdom: Animalia; phylum: Arthropoda; class: Insecta; order: Coleoptera; family: Chrysomelidae; genus: Epitrix; specificEpithet: hirtipennis; scientificNameAuthorship: Melsheimer; country: Japan; stateProvince: Kanagawa; municipality: Atsugi-shi; locality: Atsugi Campus, Tokyo University of Agriculture, Funako; minimumElevationInMeters: 49; maximumElevationInMeters: 49; decimalLatitude: 35.431707; decimalLongitude: 139.345165; geodeticDatum: WGS84; samplingProtocol: beating of leaves and branches (including visual searches); eventDate: 2016-09-28; individualCount: 4; lifeStage: adult; recordedBy: Y. Yamada; otherCatalogNumbers: 2017-00296 | 2017-00297 | 2017-00298 | 2017-00299; identifiedBy: Y. Yamada; dateldentified: 2017; institutionCode: LETUA; collectionCode: IC

c. namePublishedln: 1847; kingdom: Animalia; phylum: Arthropoda; class: Insecta; order: Coleoptera; family: Chrysomelidae; genus: Epitrix; specificEpithet: hirtipennis; scientificNameAuthorship: Melsheimer; country: Japan; stateProvince: Kanagawa; municipality: Atsugi-shi; locality: Atsugi Campus, Tokyo University of Agriculture, Funako; minimumElevationInMeters: 49; maximumElevationInMeters: 49; decimalLatitude: 35.431707; decimalLongitude: 139.345165; geodeticDatum: WGS84; samplingProtocol: beating of leaves and branches (including visual searches); eventDate: 2016-09-29; individualCount: 5; lifeStage: adult; recordedBy: Y. Yamada; otherCatalogNumbers: 2017-00300 | 2017-00301 | 2017-00302 | 2017-00303 | 2017-00304; identifiedBy: Y. Yamada; dateldentified: 2017; institutionCode: LETUA; collectionCode: IC

d. namePublishedln: 1847; kingdom: Animalia; phylum: Arthropoda; class: Insecta; order: Coleoptera; family: Chrysomelidae; genus: Epitrix; specificEpithet: hirtipennis; scientificNameAuthorship: Melsheimer; country: Japan; stateProvince: Kanagawa; municipality: Atsugi-shi; locality: Atsugi Campus, Tokyo University of Agriculture, Funako; minimumElevationInMeters: 49; maximumElevationInMeters: 49; decimalLatitude: 35.431707; decimalLongitude: 139.345165; geodeticDatum: WGS84; samplingProtocol: beating of leaves and branches (including visual searches); eventDate: 2016-09-30; individualCount: 8; lifeStage: adult; recordedBy: Y. Yamada; otherCatalogNumbers: 2017-00305 | 2017-00306 | 2017-00307 | 2017-00308 | 2017-00309 | 2017-00310 | 2017-00311 | 2017-00312; identifiedBy: Y. Yamada; dateldentified: 2017; institutionCode: LETUA; collectionCode: IC

e. namePublishedln: 1847; kingdom: Animalia; phylum: Arthropoda; class: Insecta; order: Coleoptera; family: Chrysomelidae; genus: Epitrix; specificEpithet: hirtipennis; scientificNameAuthorship: Melsheimer; country: Japan; stateProvince: Kanagawa; municipality: Atsugi-shi; locality: Atsugi Campus, Tokyo University of Agriculture, Funako; minimumElevationInMeters: 49; maximumElevationInMeters: 49; decimalLatitude: 35.431707; decimalLongitude: 139.345165; geodeticDatum: WGS84; samplingProtocol: beating of leaves and branches (including visual searches); eventDate: 2016-10-01; individualCount: 7; lifeStage: adult; recordedBy: Y. Yamada; otherCatalogNumbers: 2017-00313 | 2017-00314 | 2017-00315 | 2017-00316 | 2017-00317 | 2017-00318 | 2017-00319; identifiedBy: Y. Yamada; dateldentified: 2017; institutionCode: LETUA; collectionCode: IC

f. namePublishedln: 1847; kingdom: Animalia; phylum: Arthropoda; class: Insecta; order: Coleoptera; family: Chrysomelidae; genus: Epitrix; specificEpithet: hirtipennis; scientificNameAuthorship: Melsheimer; country: Japan; stateProvince: Kanagawa; 
municipality: Atsugi-shi; locality: Atsugi Campus, Tokyo University of Agriculture, Funako; minimumElevationInMeters: 49; maximumElevationInMeters: 49; decimalLatitude: 35.431707; decimalLongitude: 139.345165; geodeticDatum: WGS84; samplingProtocol: beating of leaves and branches (including visual searches); eventDate: 2016-10-04; individualCount: 4; lifeStage: adult; recordedBy: Y. Yamada; otherCatalogNumbers: 2017-00320 | 2017-00321 | 2017-00322 | 2017-00323; identifiedBy: Y. Yamada; dateldentified: 2017; institutionCode: LETUA; collectionCode: IC

g. namePublishedln: 1847; kingdom: Animalia; phylum: Arthropoda; class: Insecta; order: Coleoptera; family: Chrysomelidae; genus: Epitrix; specificEpithet: hirtipennis; scientificNameAuthorship: Melsheimer; country: Japan; stateProvince: Kanagawa; municipality: Atsugi-shi; locality: Atsugi Campus, Tokyo University of Agriculture, Funako; minimumElevationInMeters: 49; maximumElevationInMeters: 49; decimalLatitude: 35.431707; decimalLongitude: 139.345165; geodeticDatum: WGS84; samplingProtocol: beating of leaves and branches (including visual searches); eventDate: 2016-10-14; individualCount: 4; lifeStage: adult; recordedBy: Y. Yamada; otherCatalogNumbers: 2017-00324 | 2017-00325 | 2017-00326 | 2017-00327; identifiedBy: Y. Yamada; dateldentified: 2017; institutionCode: LETUA; collectionCode: IC

h. namePublishedln: 1847; kingdom: Animalia; phylum: Arthropoda; class: Insecta; order: Coleoptera; family: Chrysomelidae; genus: Epitrix; specificEpithet: hirtipennis; scientificNameAuthorship: Melsheimer; country: Japan; stateProvince: Kanagawa; municipality: Atsugi-shi; locality: Atsugi Campus, Tokyo University of Agriculture, Funako; minimumElevationInMeters: 49; maximumElevationInMeters: 49; decimalLatitude: 35.431707; decimalLongitude: 139.345165; geodeticDatum: WGS84; samplingProtocol: beating of leaves and branches (including visual searches); eventDate: 2016-10-15; individualCount: 4; lifeStage: adult; recordedBy: Y. Yamada; otherCatalogNumbers: 2017-00328 | 2017-00329 | 2017-00330 | 2017-00331; identifiedBy: Y. Yamada; dateldentified: 2017; institutionCode: LETUA; collectionCode: IC

i. namePublishedln: 1847; kingdom: Animalia; phylum: Arthropoda; class: Insecta; order: Coleoptera; family: Chrysomelidae; genus: Epitrix; specificEpithet: hirtipennis; scientificNameAuthorship: Melsheimer; country: Japan; stateProvince: Kanagawa; municipality: Atsugi-shi; locality: Atsugi Campus, Tokyo University of Agriculture, Funako; minimumElevationInMeters: 49; maximumElevationInMeters: 49; decimalLatitude: 35.431707; decimalLongitude: 139.345165; geodeticDatum: WGS84; samplingProtocol: beating of leaves and branches (including visual searches); eventDate: 2016-10-18; individualCount: 8; lifeStage: adult; recordedBy: Y. Yamada; otherCatalogNumbers: 2017-00332 | 2017-00333 | 2017-00334 | 2017-00335 | 2017-00336 | 2017-00337 | 2017-00338 | 2017-00339; identifiedBy: Y. Yamada; dateldentified: 2017; institutionCode: LETUA; collectionCode: IC

j. namePublishedln: 1847; kingdom: Animalia; phylum: Arthropoda; class: Insecta; order: Coleoptera; family: Chrysomelidae; genus: Epitrix; specificEpithet: hirtipennis; scientificNameAuthorship: Melsheimer; country: Japan; stateProvince: Kanagawa; municipality: Atsugi-shi; locality: Atsugi Campus, Tokyo University of Agriculture, Funako; minimumElevationInMeters: 49; maximumElevationInMeters: 49; decimalLatitude: 35.431707; decimalLongitude: 139.345165; geodeticDatum: WGS84; samplingProtocol: beating of leaves and branches (including visual searches); eventDate: 2016-10-20; individualCount: 1; lifeStage: adult; recordedBy: Y. Yamada; otherCatalogNumbers: 2017-00340; identifiedBy: Y. Yamada; dateldentified: 2017; institutionCode: LETUA; collectionCode: IC

k. namePublishedln: 1847; kingdom: Animalia; phylum: Arthropoda; class: Insecta; order: Coleoptera; family: Chrysomelidae; genus: Epitrix; specificEpithet: hirtipennis; 
scientificNameAuthorship: Melsheimer; country: Japan; stateProvince: Kanagawa; municipality: Atsugi-shi; locality: Atsugi Campus, Tokyo University of Agriculture, Funako; minimumElevationInMeters: 49; maximumElevationInMeters: 49; decimalLatitude: 35.431707; decimalLongitude: 139.345165; geodeticDatum: WGS84; samplingProtocol: beating of leaves and branches (including visual searches); eventDate: 2016-10-24; individualCount: 1; lifeStage: adult; recordedBy: Y. Yamada; otherCatalogNumbers: 2017-00341; identifiedBy: Y. Yamada; dateldentified: 2017; institutionCode: LETUA; collectionCode: IC

I. namePublishedln: 1847; kingdom: Animalia; phylum: Arthropoda; class: Insecta; order: Coleoptera; family: Chrysomelidae; genus: Epitrix; specificEpithet: hirtipennis; scientificNameAuthorship: Melsheimer; country: Japan; stateProvince: Kanagawa; municipality: Atsugi-shi; locality: Atsugi Campus, Tokyo University of Agriculture, Funako; minimumElevationInMeters: 49; maximumElevationInMeters: 49; decimalLatitude: 35.431707; decimalLongitude: 139.345165; geodeticDatum: WGS84; samplingProtocol: beating of leaves and branches (including visual searches); eventDate: 2016-11-03; individualCount: 4; lifeStage: adult; recordedBy: Y. Yamada; otherCatalogNumbers: 2017-00342 | 2017-00343 | 2017-00344 | 2017-00345; identifiedBy: Y. Yamada; dateldentified: 2017; institutionCode: LETUA; collectionCode: IC

m. namePublishedln: 1847; kingdom: Animalia; phylum: Arthropoda; class: Insecta; order: Coleoptera; family: Chrysomelidae; genus: Epitrix; specificEpithet: hirtipennis; scientificNameAuthorship: Melsheimer; country: Japan; stateProvince: Kanagawa; municipality: Atsugi-shi; locality: Atsugi Campus, Tokyo University of Agriculture, Funako; minimumElevationInMeters: 49; maximumElevationInMeters: 49; decimalLatitude: 35.431707; decimalLongitude: 139.345165; geodeticDatum: WGS84; samplingProtocol: beating of leaves and branches (including visual searches); eventDate: 2016-11-08; individualCount: 2; lifeStage: adult; recordedBy: Y. Yamada; otherCatalogNumbers: 2017-00346 | 2017-00347; identifiedBy: Y. Yamada; dateldentified: 2017; institutionCode: LETUA; collectionCode: IC

n. namePublishedln: 1847; kingdom: Animalia; phylum: Arthropoda; class: Insecta; order: Coleoptera; family: Chrysomelidae; genus: Epitrix; specificEpithet: hirtipennis; scientificNameAuthorship: Melsheimer; country: Japan; stateProvince: Kanagawa; municipality: Atsugi-shi; locality: Atsugi Campus, Tokyo University of Agriculture, Funako; minimumElevationInMeters: 49; maximumElevationInMeters: 49; decimalLatitude: 35.431707; decimalLongitude: 139.345165; geodeticDatum: WGS84; samplingProtocol: beating of leaves and branches (including visual searches); eventDate: 2016-11-10; individualCount: 1; lifeStage: adult; recordedBy: Y. Yamada; otherCatalogNumbers: 2017-00348; identifiedBy: Y. Yamada; dateldentified: 2017; institutionCode: LETUA; collectionCode: IC

o. namePublishedln: 1847; kingdom: Animalia; phylum: Arthropoda; class: Insecta; order: Coleoptera; family: Chrysomelidae; genus: Epitrix; specificEpithet: hirtipennis; scientificNameAuthorship: Melsheimer; country: Japan; stateProvince: Kanagawa; municipality: Atsugi-shi; locality: Atsugi Campus, Tokyo University of Agriculture, Funako; minimumElevationInMeters: 49; maximumElevationInMeters: 49; decimalLatitude: 35.431707; decimalLongitude: 139.345165; geodeticDatum: WGS84; samplingProtocol: beating of leaves and branches (including visual searches); eventDate: 2016-11-15; individualCount: 1; lifeStage: adult; recordedBy: Y. Yamada; otherCatalogNumbers: 2017-00349; identifiedBy: Y. Yamada; dateldentified: 2017; institutionCode: LETUA; collectionCode: IC

p. namePublishedln: 1847; kingdom: Animalia; phylum: Arthropoda; class: Insecta; order: Coleoptera; family: Chrysomelidae; genus: Epitrix; specificEpithet: hirtipennis; 
scientificNameAuthorship: Melsheimer; country: Japan; stateProvince: Kanagawa; municipality: Atsugi-shi; locality: Atsugi Campus, Tokyo University of Agriculture, Funako; minimumElevationInMeters: 49; maximumElevationInMeters: 49; decimalLatitude: 35.431707; decimalLongitude: 139.345165; geodeticDatum: WGS84; samplingProtocol: beating of leaves and branches (including visual searches); eventDate: 2016-11-17; individualCount: 1; lifeStage: adult; recordedBy: Y. Yamada; otherCatalogNumbers: 2017-00350; identifiedBy: Y. Yamada; dateldentified: 2017; institutionCode: LETUA; collectionCode: IC

q. namePublishedln: 1847; kingdom: Animalia; phylum: Arthropoda; class: Insecta; order: Coleoptera; family: Chrysomelidae; genus: Epitrix; specificEpithet: hirtipennis; scientificNameAuthorship: Melsheimer; country: Japan; stateProvince: Kanagawa; municipality: Atsugi-shi; locality: Atsugi Campus, Tokyo University of Agriculture, Funako; minimumElevationInMeters: 49; maximumElevationInMeters: 49; decimalLatitude: 35.431707; decimalLongitude: 139.345165; geodeticDatum: WGS84; samplingProtocol: beating of leaves and branches (including visual searches); eventDate: 2016-11-25; individualCount: 2; lifeStage: adult; recordedBy: Y. Yamada; otherCatalogNumbers: 2017-00351 | 2017-00352; identifiedBy: Y. Yamada; dateldentified: 2017; institutionCode: LETUA; collectionCode: IC

Notes: Known as a recent alien species to Japan (Harada and Takizawa 2012)

\section{Order Lepidoptera Linnaeus, 1758}

\section{Family Noctuidae Latreille, 1809}

\section{Spodoptera litura (Fabricius, 1775)}

\section{Materials}

a. namePublishedln: 1775; kingdom: Animalia; phylum: Arthropoda; class: Insecta; order: Lepidoptera; family: Noctuidae; genus: Spodoptera; specificEpithet: litura; scientificNameAuthorship: Fabricius; country: Japan; stateProvince: Kanagawa; municipality: Atsugi-shi; locality: Atsugi Campus, Tokyo University of Agriculture, Funako; minimumElevationInMeters: 49; maximumElevationInMeters: 49; decimalLatitude: 35.431707; decimalLongitude: 139.345165; geodeticDatum: WGS84; samplingProtocol: beating of leaves and branches (including visual searches); eventDate: 2016-10-24; individualCount: 1; lifeStage: larva; recordedBy: Y. Yamada; otherCatalogNumbers: 2017-00353; identifiedBy: T. Ishikawa; dateldentified: 2017; institutionCode: LETUA; collectionCode: IC

b. namePublishedln: 1775; kingdom: Animalia; phylum: Arthropoda; class: Insecta; order: Lepidoptera; family: Noctuidae; genus: Spodoptera; specificEpithet: litura; scientificNameAuthorship: Fabricius; country: Japan; stateProvince: Kanagawa; municipality: Atsugi-shi; locality: Atsugi Campus, Tokyo University of Agriculture, Funako; minimumElevationInMeters: 27; maximumElevationInMeters: 27; decimalLatitude: 35.43043; decimalLongitude: 139.349516; geodeticDatum: WGS84; samplingProtocol: beating of leaves and branches (including visual searches); eventDate: 2016-11-18; individualCount: 1; lifeStage: larva; recordedBy: Y. Yamada; otherCatalogNumbers: 2017-00354; identifiedBy: T. Ishikawa; dateldentified: 2017; institutionCode: LETUA; collectionCode: IC 


\section{Trichoplusia ni (Hübner, 1803)}

\section{Materials}

a. namePublishedln: 1803; kingdom: Animalia; phylum: Arthropoda; class: Insecta; order: Lepidoptera; family: Noctuidae; genus: Trichoplusia; specificEpithet: ni; scientificNameAuthorship: Hübner; country: Japan; stateProvince: Kanagawa; municipality: Atsugi-shi; locality: Atsugi Campus, Tokyo University of Agriculture, Funako; minimumElevationInMeters: 27; maximumElevationInMeters: 27; decimalLatitude: 35.43043; decimalLongitude: 139.349516; geodeticDatum: WGS84; samplingProtocol: beating of leaves and branches (including visual searches); eventDate: 2016-11-08; individualCount: 1; lifeStage: larva; recordedBy: K. Niwa; otherCatalogNumbers: 2017-00355; identifiedBy: T. Ishikawa; dateldentified: 2017; institutionCode: LETUA; collectionCode: IC

b. namePublishedln: 1803; kingdom: Animalia; phylum: Arthropoda; class: Insecta; order: Lepidoptera; family: Noctuidae; genus: Trichoplusia; specificEpithet: ni; scientificNameAuthorship: Hübner; country: Japan; stateProvince: Kanagawa; municipality: Atsugi-shi; locality: Atsugi Campus, Tokyo University of Agriculture, Funako; minimumElevationInMeters: 27; maximumElevationInMeters: 27; decimalLatitude: 35.43043; decimalLongitude: 139.349516; geodeticDatum: WGS84; samplingProtocol: beating of leaves and branches (including visual searches); eventDate: 2016-11-18; individualCount: 8; lifeStage: larva; recordedBy: K. Niwa; otherCatalogNumbers: 2017-00356 | 2017-00357 | 2017-00358 | 2017-00359 | 2017-00360 | 2017-00361 | 2017-00362 | 2017-00363; identifiedBy: T. Ishikawa; dateldentified: 2017; institutionCode: LETUA; collectionCode: IC

\section{Order Diptera Linnaeus, 1758}

\section{Family Agromyzidae Fallén, 1823}

\section{Liriomyza sativae Blanchard, 1938}

\section{Materials}

a. namePublishedln: 1938; kingdom: Animalia; phylum: Arthropoda; class: Insecta; order: Diptera; family: Agromyzidae; genus: Liriomyza; specificEpithet: sativae; scientificNameAuthorship: Blanchard; country: Japan; stateProvince: Kanagawa; municipality: Atsugi-shi; locality: Atsugi Campus, Tokyo University of Agriculture, Funako; minimumElevationInMeters: 42; maximumElevationInMeters: 42; decimalLatitude: 35.428874; decimalLongitude: 139.34929; geodeticDatum: WGS84; samplingProtocol: beating of leaves and branches (including visual searches); eventDate: 2016-11-17; individualCount: 4; lifeStage: adult; recordedBy: O-k. Kim; otherCatalogNumbers: 2017-00364 | 2017-00365 | 2017-00366 | 2017-00367; identifiedBy: Y. Yamada; dateldentified: 2017; institutionCode: LETUA; collectionCode: IC

b. namePublishedln: 1938; kingdom: Animalia; phylum: Arthropoda; class: Insecta; order: Diptera; family: Agromyzidae; genus: Liriomyza; specificEpithet: sativae; scientificNameAuthorship: Blanchard; country: Japan; stateProvince: Kanagawa; municipality: Atsugi-shi; locality: Atsugi Campus, Tokyo University of Agriculture, Funako; minimumElevationInMeters: 42; maximumElevationInMeters: 42; decimalLatitude: 
35.428874; decimalLongitude: 139.34929; geodeticDatum: WGS84; samplingProtocol: beating of leaves and branches (including visual searches); eventDate: 2016-12-15; individualCount: 5; lifeStage: adult; recordedBy: Y. Yamada; otherCatalogNumbers: 2017-00368 | 2017-00369 | 2017-00370 | 2017-00371 | 2017-00372; identifiedBy: Y. Yamada; dateldentified: 2017; institutionCode: LETUA; collectionCode: IC

c. namePublishedln: 1938; kingdom: Animalia; phylum: Arthropoda; class: Insecta; order: Diptera; family: Agromyzidae; genus: Liriomyza; specificEpithet: sativae; scientificNameAuthorship: Blanchard; country: Japan; stateProvince: Kanagawa; municipality: Atsugi-shi; locality: Atsugi Campus, Tokyo University of Agriculture, Funako; minimumElevationInMeters: 42; maximumElevationInMeters: 42; decimalLatitude: 35.428874; decimalLongitude: 139.34929; geodeticDatum: WGS84; samplingProtocol: beating of leaves and branches (including visual searches); eventDate: 2016-12-19; individualCount: 1; lifeStage: adult; recordedBy: Y. Yamada; otherCatalogNumbers: 2017-00373; identifiedBy: Y. Yamada; dateldentified: 2017; institutionCode: LETUA; collectionCode: IC

d. namePublishedln: 1938; kingdom: Animalia; phylum: Arthropoda; class: Insecta; order: Diptera; family: Agromyzidae; genus: Liriomyza; specificEpithet: sativae; scientificNameAuthorship: Blanchard; country: Japan; stateProvince: Kanagawa; municipality: Atsugi-shi; locality: Atsugi Campus, Tokyo University of Agriculture, Funako; minimumElevationInMeters: 42; maximumElevationInMeters: 42; decimalLatitude: 35.428874; decimalLongitude: 139.34929; geodeticDatum: WGS84; samplingProtocol: beating of leaves and branches (including visual searches); eventDate: 2017-01-07; individualCount: 13; lifeStage: adult; recordedBy: Y. Yamada; otherCatalog Numbers: | | 2017-00386; identifiedBy: Y. Yamada; dateldentified: 2017; institutionCode: LETUA; collectionCode: IC

\title{
Class Arachnida Lamarck, 1801
}

\author{
Order Acari Leach, 1817
}

\section{Family Tarsonemidae Kramer, 1877}

\section{Polyphagotarsonemus latus (Banks, 1904)}

\section{Material}

a. namePublishedln: 1904; kingdom: Animalia; phylum: Arthropoda; class: Arachnida; order: Acari; family: Tarsonemidae; genus: Polyphagotarsonemus; specificEpithet: latus; scientificNameAuthorship: Banks; country: Japan; stateProvince: Kanagawa; municipality: Atsugi-shi; locality: Atsugi Campus, Tokyo University of Agriculture, Funako; minimumElevationInMeters: 42; maximumElevationInMeters: 42; decimalLatitude: 35.428874; decimalLongitude: 139.34929; geodeticDatum: WGS84; samplingProtocol: beating of leaves and branches (including visual searches); eventDate: 2017-01-07; individualCount: 1; lifeStage: adult; recordedBy: Y. Yamada; otherCatalogNumbers: 2017-00387; identifiedBy: Y. Yamada; dateldentified: 2017; institutionCode: LETUA; collectionCode: IC 


\section{Family Tetranychidae Donnadieu, 1875}

\section{Tetranychus urticae Koch, 1836}

\section{Materials}

a. namePublishedln: 1836; kingdom: Animalia; phylum: Arthropoda; class: Arachnida; order: Acari; family: Tetranychidae; genus: Tetranychus; specificEpithet: urticae; scientificNameAuthorship: Koch; country: Japan; stateProvince: Kanagawa; municipality: Atsugi-shi; locality: Atsugi Campus, Tokyo University of Agriculture, Funako; minimumElevationInMeters: 49; maximumElevationInMeters: 49; decimalLatitude: 35.431707; decimalLongitude: 139.345165; geodeticDatum: WGS84; samplingProtocol: beating of leaves and branches (including visual searches); eventDate: 2016-08-30; individualCount: 8; lifeStage: adult; recordedBy: T. Ishikawa; otherCatalogNumbers: 2017-00388 | 2017-00389 | 2017-00390 | 2017-00391 | 2017-00392 | 2017-00393 | 2017-00394 | 2017-00395; identifiedBy: Y. Yamada; dateldentified: 2017; institutionCode: LETUA; collectionCode: IC

b. namePublishedln: 1836; kingdom: Animalia; phylum: Arthropoda; class: Arachnida; order: Acari; family: Tetranychidae; genus: Tetranychus; specificEpithet: urticae; scientificNameAuthorship: Koch; country: Japan; stateProvince: Kanagawa; municipality: Atsugi-shi; locality: Atsugi Campus, Tokyo University of Agriculture, Funako; minimumElevationInMeters: 42; maximumElevationInMeters: 42; decimalLatitude: 35.428874; decimalLongitude: 139.34929; geodeticDatum: WGS84; samplingProtocol: beating of leaves and branches (including visual searches); eventDate: 2017-01-07; individualCount: 13; lifeStage: adult; recordedBy: Y. Yamada; otherCatalogNumbers: 2017-00396 | 2017-00397 | 2017-00398 | 2017-00399 | 2017-00400 | 2017-00401 | 2017-00402 | 2017-00403 | 2017-00404 | 2017-00405 | 2017-00406 | 2017-00407 | 2017-00408; identifiedBy: Y. Yamada; dateldentified: 2017; institutionCode: LETUA; collectionCode: IC

c. namePublishedln: 1836; kingdom: Animalia; phylum: Arthropoda; class: Arachnida; order: Acari; family: Tetranychidae; genus: Tetranychus; specificEpithet: urticae; scientificNameAuthorship: Koch; country: Japan; stateProvince: Kanagawa; municipality: Atsugi-shi; locality: Atsugi Campus, Tokyo University of Agriculture, Funako; minimumElevationInMeters: 42; maximumElevationInMeters: 42; decimalLatitude: 35.428874; decimalLongitude: 139.34929; geodeticDatum: WGS84; samplingProtocol: beating of leaves and branches (including visual searches); eventDate: 2017-01-09; individualCount: 18; lifeStage: adult; recordedBy: Y. Yamada; otherCatalogNumbers: 2017-00409 | 2017-00410 | 2017-00411 | 2017-00412 | 2017-00413 | 2017-00414 | | 2017-00421 | 2017-00422 | 2017-00423 | 2017-00424 | 2017-00425 | 2017-00426; identifiedBy: Y. Yamada; dateldentified: 2017; institutionCode: LETUA; collectionCode: IC d. namePublishedln: 1836; kingdom: Animalia; phylum: Arthropoda; class: Arachnida; order: Acari; family: Tetranychidae; genus: Tetranychus; specificEpithet: urticae; scientificNameAuthorship: Koch; country: Japan; stateProvince: Kanagawa; municipality: Atsugi-shi; locality: Atsugi Campus, Tokyo University of Agriculture, Funako; minimumElevationInMeters: 42; maximumElevationInMeters: 42; decimalLatitude: 35.428874; decimalLongitude: 139.34929; geodeticDatum: WGS84; samplingProtocol: beating of leaves and branches (including visual searches); eventDate: 2017-01-09; individualCount: 2; lifeStage: larva; recordedBy: Y. Yamada; otherCatalogNumbers: 
2017-00427 | 2017-00428; identifiedBy: Y. Yamada; dateldentified: 2017; institutionCode: LETUA; collectionCode: IC

e. namePublishedln: 1836; kingdom: Animalia; phylum: Arthropoda; class: Arachnida; order: Acari; family: Tetranychidae; genus: Tetranychus; specificEpithet: urticae; scientificNameAuthorship: Koch; country: Japan; stateProvince: Kanagawa; municipality: Atsugi-shi; locality: Atsugi Campus, Tokyo University of Agriculture, Funako; minimumElevationInMeters: 42; maximumElevationInMeters: 42; decimalLatitude: 35.428874; decimalLongitude: 139.34929; geodeticDatum: WGS84; samplingProtocol: beating of leaves and branches (including visual searches); eventDate: 2017-01-12; individualCount: 15; lifeStage: adult; recordedBy: Y. Yamada; otherCatalogNumbers: 2017-00429 | 2017-00430 | 2017-00431 | 2017-00432 | 2017-00433 | 2017-00434 | | 2017-00441 | 2017-00442 | 2017-00443; identifiedBy: Y. Yamada; dateldentified: 2017; institutionCode: LETUA; collectionCode: IC

f. namePublishedln: 1836; kingdom: Animalia; phylum: Arthropoda; class: Arachnida; order: Acari; family: Tetranychidae; genus: Tetranychus; specificEpithet: urticae; scientificNameAuthorship: Koch; country: Japan; stateProvince: Kanagawa; municipality: Atsugi-shi; locality: Atsugi Campus, Tokyo University of Agriculture, Funako; minimumElevationInMeters: 42; maximumElevationInMeters: 42; decimalLatitude: 35.428874; decimalLongitude: 139.34929; geodeticDatum: WGS84; samplingProtocol: beating of leaves and branches (including visual searches); eventDate: 2017-01-16; individualCount: 8; lifeStage: adult; recordedBy: Y. Yamada; otherCatalogNumbers: 2017-00444 | 2017-00445 | 2017-00446 | 2017-00447 | 2017-00448 | 2017-00449 | 2017-00450 | 2017-00451; identifiedBy: Y. Yamada; dateldentified: 2017; institutionCode: LETUA; collectionCode: IC

g. namePublishedln: 1836; kingdom: Animalia; phylum: Arthropoda; class: Arachnida; order: Acari; family: Tetranychidae; genus: Tetranychus; specificEpithet: urticae; scientificNameAuthorship: Koch; country: Japan; stateProvince: Kanagawa; municipality: Atsugi-shi; locality: Atsugi Campus, Tokyo University of Agriculture, Funako; minimumElevationInMeters: 42; maximumElevationInMeters: 42; decimalLatitude: 35.428874; decimalLongitude: 139.34929; geodeticDatum: WGS84; samplingProtocol: beating of leaves and branches (including visual searches); eventDate: 2017-01-21; individualCount: 12; lifeStage: adult; recordedBy: Y. Yamada; otherCatalogNumbers: 2017-00452 | 2017-00453 | 2017-00454 | 2017-00455 | 2017-00456 | 2017-00457 | 2017-00458 | 2017-00459 | 2017-00460 | 2017-00461 | 2017-00462 | 2017-00463; identifiedBy: Y. Yamada; dateldentified: 2017; institutionCode: LETUA; collectionCode: IC

\section{Discussion}

Prior to the present study, nine groups of insects and mites have been known as pests of pepino plants in Japan (Table 3), including those that are inadequately identified, such as Helicoverpa spp. at the genus level, aphids at the family level (Aphididae), and mites at the order level (Acari). Among these, three groups were identified at the species level, comprising the greenhouse whitefly Trialeurodes vaporariorum (Westwood, 1856), the potato tuberworm Phthorimaea operculella (Zeller, 1873), and the two-spotted spider mite Tetranychus urticae Koch, 1836. In the present study, our survey in the campus revealed the presence of 11 species of insect and mite pests on pepino as mentioned above. Only $T$. urticae is common in the previous records as well as the results of the present study; in 
addition, $T$. vaporariorum and $P$. operculella were not found in our survey. Currently, 13 species of insects and mites are recognized as pests of pepino plants in Japan; all of these are also the pests of major solanaceous crops such as tomato, eggplant, green pepper, and potato (The Japanese Society of Applied Entomology and Zoology 2006). It is likely that $T$. vaporariorum and $P$. operculella may be detected on pepino from the campus, because the two species are widely distributed in Kanagawa Prefecture (Matsumoto 2004, Nakajima and Yamamoto 2004) where our study was carried out.

Table 3.

Insect and mite pests of pepino plants previously recorded in Japan.

\begin{tabular}{|c|c|c|c|c|c|}
\hline Class & Order & Family & $\begin{array}{l}\text { Group (of } \\
\text { species and its } \\
\text { allies) }\end{array}$ & Species & References \\
\hline Insecta & Hemiptera & Aleyrodidae & - & $\begin{array}{l}\text { Trialeurodes } \\
\text { vaporariorum } \\
\text { (Westwood, 1856) }\end{array}$ & $\begin{array}{l}\text { Furusato (1984), Takahashi } \\
\text { (1985), Takagi (1985), Kita } \\
\text { (1986), Odagiri et al. (1986), } \\
\text { Ozawa (1986) }\end{array}$ \\
\hline Insecta & Hemiptera & Apididae & aphids & - & $\begin{array}{l}\text { Takagi (1985), Takahashi (1985), } \\
\text { Kita (1986), Odagiri et al. (1986), } \\
\text { Ozawa (1986), Takahashi (1986) }\end{array}$ \\
\hline Insecta & Lepidoptera & Noctuidae & $\begin{array}{l}\text { Helicoverpa } \\
\text { spp. }\end{array}$ & - & Odagiri et al. (1986) \\
\hline Insecta & Lepidoptera & Noctuidae & Spodoptera spp. & - & Odagiri et al. (1986) \\
\hline Insecta & Lepidoptera & Gelechiidae & - & $\begin{array}{l}\text { Phthorimaea } \\
\text { operculella (Zeller, } \\
1873 \text { ) }\end{array}$ & Ozawa (1986) \\
\hline Insecta & Lepidoptera & unspecified & $\begin{array}{l}\text { green } \\
\text { caterpillars }\end{array}$ & - & Furusato (1986) \\
\hline Arachnida & Acari & unspecified & mites & - & $\begin{array}{l}\text { Furusato (1984), Kita (1986), } \\
\text { Odagiri et al. (1986) }\end{array}$ \\
\hline Arachnida & Acari & Tetranychidae & spider mites & - & $\begin{array}{l}\text { Takahashi (1985), Takagi (1985), } \\
\text { Ozawa (1986), Takahashi (1986) }\end{array}$ \\
\hline Arachnida & Acari & Tetranychidae & - & $\begin{array}{l}\text { Tetranychus urticae } \\
\text { Koch, } 1836\end{array}$ & Ozawa (1986) \\
\hline
\end{tabular}

Currently, 25 species of insects and mites have been reported as pests of pepino plants worldwide (Table 4) (Galbreath and Clearwater 1983, Larraín 2002, Akyazi 2012). Among these pests, 16 species are distributed in Japan, but only three species, Trichoplusia ni, Polyphagotarsonemus latus, and Tetranychus urticae, were evaluated as pests of pepino in the present study. This indicates that the remaining 13 species are very likely to be 
potential pests on pepino in Japan. Therefore, at least 26 insect and mite species, including the 13 currently known and the 13 potential ones in Japan, will be recognized as pests of pepino in the near future if the cultivation of pepinos spreads throughout the Japanese Archipelago.

\section{Table 4.}

Insect and mite pests of pepino plants previously recorded worldwide (excluding Japan).

\begin{tabular}{|c|c|c|c|c|c|c|}
\hline Class & Order & Family & Species & $\begin{array}{l}\text { Country } \\
\text { recorded } \\
\text { as a pest }\end{array}$ & References & Notes \\
\hline Insecta & Orthoptera & Acrididae & $\begin{array}{l}\text { Schistocerca cancellata } \\
\text { (Serville, 1838) }\end{array}$ & Chili & $\begin{array}{l}\text { Larraín } \\
\text { (2002) }\end{array}$ & \\
\hline Insecta & Thysanoptera & Thripidae & $\begin{array}{l}\text { Frankliniella occidentalis } \\
\text { (Pergande, 1895) }\end{array}$ & Chili & $\begin{array}{l}\text { Larraín } \\
\text { (2002) }\end{array}$ & $\begin{array}{l}\text { Distributed in } \\
\text { Japan }\end{array}$ \\
\hline Insecta & Thysanoptera & Thripidae & $\begin{array}{l}\text { Thrips tabaci Lindeman, } \\
1889\end{array}$ & Chili & $\begin{array}{l}\text { Larraín } \\
\text { (2002) }\end{array}$ & $\begin{array}{l}\text { Distributed in } \\
\text { Japan }\end{array}$ \\
\hline Insecta & Hemiptera & Aphididae & $\begin{array}{l}\text { Aulacorthum solani } \\
\text { (Kaltenbach, 1843) }\end{array}$ & Chili & $\begin{array}{l}\text { Larraín } \\
\text { (2002) }\end{array}$ & $\begin{array}{l}\text { Distributed in } \\
\text { Japan }\end{array}$ \\
\hline Insecta & Hemiptera & Aphididae & $\begin{array}{l}\text { Macrosiphum } \\
\text { euphorbiae (Thomas, } \\
1878 \text { ) }\end{array}$ & Chili & $\begin{array}{l}\text { Larraín } \\
\text { (2002) }\end{array}$ & $\begin{array}{l}\text { Distributed in } \\
\text { Japan }\end{array}$ \\
\hline Insecta & Hemiptera & Aphididae & $\begin{array}{l}\text { Myzus persicae (Sulzer, } \\
1776)\end{array}$ & Chili & $\begin{array}{l}\text { Larraín } \\
\text { (2002) }\end{array}$ & $\begin{array}{l}\text { Distributed in } \\
\text { Japan }\end{array}$ \\
\hline Insecta & Hemiptera & Pseudococcidae & $\begin{array}{l}\text { Phenacoccus } \\
\text { solenopsis Tinsley, } 1898\end{array}$ & Chili & $\begin{array}{l}\text { Larraín } \\
\text { (2002) }\end{array}$ & $\begin{array}{l}\text { Distributed in } \\
\text { Japan }\end{array}$ \\
\hline Insecta & Hemiptera & Pseudococcidae & $\begin{array}{l}\text { Pseudococcus viburni } \\
\text { (Signoret, 1875) }\end{array}$ & Chili & $\begin{array}{l}\text { Larraín } \\
\text { (2002) }\end{array}$ & $\begin{array}{l}\text { Distributed in } \\
\text { Japan }\end{array}$ \\
\hline Insecta & Hemiptera & Psyllidae & $\begin{array}{l}\text { Russelliana solanicola } \\
\text { Tuthill, } 1959\end{array}$ & Chili & $\begin{array}{l}\text { Larraín } \\
\text { (2002) }\end{array}$ & \\
\hline Insecta & Hemiptera & Triozidae & $\begin{array}{l}\text { Trioza chenopodii } \\
\text { Reuter, } 1876\end{array}$ & Chili & $\begin{array}{l}\text { Larraín } \\
\text { (2002) }\end{array}$ & $\begin{array}{l}\text { Distributed in } \\
\text { Japan }\end{array}$ \\
\hline Insecta & Hemiptera & Cicadellidae & $\begin{array}{l}\text { Xerophloea viridis } \\
\text { (Fabricius, 1794) }\end{array}$ & Chili & $\begin{array}{l}\text { Larraín } \\
\text { (2002) }\end{array}$ & \\
\hline Insecta & Hemiptera & Cicadellidae & $\begin{array}{l}\text { Paratanus exitiosus } \\
\text { (Beamer, 1943) }\end{array}$ & Chili & $\begin{array}{l}\text { Larraín } \\
\text { (2002) }\end{array}$ & \\
\hline Insecta & Diptera & Agromyzidae & $\begin{array}{l}\text { Liriomyza huidobrensis } \\
\text { (Blanchard, 1926) }\end{array}$ & Chili & $\begin{array}{l}\text { Larraín } \\
\text { (2002) }\end{array}$ & $\begin{array}{l}\text { Distributed in } \\
\text { Japan }\end{array}$ \\
\hline
\end{tabular}




\begin{tabular}{|c|c|c|c|c|c|c|}
\hline Insecta & Diptera & Tephritidae & $\begin{array}{l}\text { Rhagoletis nova } \\
\text { (Schiner, 1868) }\end{array}$ & Chili & $\begin{array}{l}\text { Larraín } \\
\text { (2002) }\end{array}$ & \\
\hline Insecta & Lepidoptera & Noctuidae & $\begin{array}{l}\text { Agrotis bilitura Guenée, } \\
1852\end{array}$ & Chili & $\begin{array}{l}\text { Larraín } \\
\text { (2002) }\end{array}$ & \\
\hline Insecta & Lepidoptera & Noctuidae & $\begin{array}{l}\text { Copitarsia turbata } \\
\text { (Herrich-Schaeffer, } \\
1855)\end{array}$ & Chili & $\begin{array}{l}\text { Larraín } \\
\text { (2002) }\end{array}$ & \\
\hline Insecta & Lepidoptera & Noctuidae & $\begin{array}{l}\text { Trichoplusia ni (Hübner, } \\
\text { [1803]) }\end{array}$ & Chili & $\begin{array}{l}\text { Larraín } \\
\text { (2002) }\end{array}$ & $\begin{array}{l}\text { Recognized as } \\
\text { a pest in Japan } \\
\text { by this study }\end{array}$ \\
\hline Insecta & Lepidoptera & Sphingidae & $\begin{array}{l}\text { Manduca sexta } \\
\text { (Linnaeus, 1763) }\end{array}$ & Chili & $\begin{array}{l}\text { Larraín } \\
\text { (2002) }\end{array}$ & $\begin{array}{l}\text { Distributed in } \\
\text { Japan }\end{array}$ \\
\hline Insecta & Lepidoptera & Gelechiidae & $\begin{array}{l}\text { Phthorimaea operculella } \\
\text { (Zeller, 1873) }\end{array}$ & Chili & $\begin{array}{l}\text { Larraín } \\
\text { (2002) }\end{array}$ & $\begin{array}{l}\text { Distributed in } \\
\text { Japan }\end{array}$ \\
\hline Insecta & Lepidoptera & Gelechiidae & $\begin{array}{l}\text { Symmetrischema } \\
\text { tangolias (Gyen, 1913) }\end{array}$ & Chili & $\begin{array}{l}\text { Larraín } \\
\text { (2002) }\end{array}$ & \\
\hline Insecta & Lepidoptera & Gelechiidae & $\begin{array}{l}\text { Tuta absoluta (Meyrick, } \\
\text { 1917) }\end{array}$ & Chili & $\begin{array}{l}\text { Larraín } \\
\text { (2002) }\end{array}$ & $\begin{array}{l}\text { Distributed in } \\
\text { Japan }\end{array}$ \\
\hline Insecta & Lepidoptera & Crambidae & $\begin{array}{l}\text { Sceliodes cordalis } \\
\text { (Doubleday, 1843) }\end{array}$ & $\begin{array}{l}\text { New } \\
\text { Zealand }\end{array}$ & $\begin{array}{l}\text { Galbreath } \\
\text { and } \\
\text { Clearwater } \\
(1983)\end{array}$ & \\
\hline Arachnida & Acari & Eriophyidae & $\begin{array}{l}\text { Aculops lycopersici } \\
\text { (Tryon, 1917) }\end{array}$ & $\begin{array}{l}\text { Chili, } \\
\text { Turkey }\end{array}$ & $\begin{array}{l}\text { Larraín } \\
\text { (2002), } \\
\text { Akyazi (2012) }\end{array}$ & $\begin{array}{l}\text { Distributed in } \\
\text { Japan }\end{array}$ \\
\hline Arachnida & Acari & Tarsonemidae & $\begin{array}{l}\text { Polyphagotarsonemus } \\
\text { latus (Banks, 1904) }\end{array}$ & Chili & $\begin{array}{l}\text { Larraín } \\
\text { (2002) }\end{array}$ & $\begin{array}{l}\text { Recognized as } \\
\text { a pest in Japan } \\
\text { by this study }\end{array}$ \\
\hline Arachnida & Acari & Tetranychidae & $\begin{array}{l}\text { Tetranychus urticae } \\
\text { Koch, } 1836\end{array}$ & Chili & $\begin{array}{l}\text { Larraín } \\
\text { (2002) }\end{array}$ & $\begin{array}{l}\text { Recognized as } \\
\text { a pest in Japan } \\
\text { by this study }\end{array}$ \\
\hline
\end{tabular}

The presence of pests can directly affect agricultural production and it may contribute to the transmission of plant viruses followed by economic losses. Bemisia tabaci, well-known as one of the most important whiteflies in terms of virus transmission, is widely distributed in the world, and is a vector of viruses of the genera Begomovirus, Carlavirus, Crinivirus, Ipomovirus, and Torradovirus (Navas-Castillo et al. 2011). Of these transmissible viruses, Tomato yellow leaf curl virus (TYLCV; Begomovirus) is the most devastating causal virus on tomato crops in many tropical, subtropical and temperate regions worldwide (Moriones and Navas-Castillo 2000). In Japan, TYLCV spread along with whitefly and indicated its 
dispersion throughout 38 prefectures by 2014 since the occurrence of TYLCV on tomato was first reported in Japan (Kato et al. 1998, Matsuura and Hoshino 2008, Ohnishi et al. 2016). However, there is no report of TYLCV incidence on pepino plants to date. Pepino can be regarded as a potential host for TYLCV through host adaptation or mutant as long as TYLCV-acquired vectors are present. Therefore, continuous monitoring of the distribution of both TYLCV and its vector is required. Some other species of insects and mites found on pepino plants in our research fields are also regarded as virus vectors (Table 5). Although some transmitted viruses cannot infect pepino plants, they may provide a habitat for virus vectors, which may impact crop ecosystems as well as virus-vector systems.

Table 5.

Insects or mites of pepino plants involved in virus transmission

\begin{tabular}{|c|c|c|}
\hline Insect vector & Transmissible genus or species of virus & Reference \\
\hline \multirow[t]{4}{*}{ Frankliniella intonsa } & Groundnut ringspot virus & \multirow[t]{4}{*}{ Riley et al. (2011) } \\
\hline & Impatiens necrotic spot virus & \\
\hline & Tomato chlorotic spot virus & \\
\hline & Tomato spotted wilt virus & \\
\hline \multirow[t]{5}{*}{ Bemisia tabaci } & Genus Begomovirus & \multirow[t]{5}{*}{ Navas-Castillo et al. (2011) } \\
\hline & Genus Carlavirus & \\
\hline & Genus Crinivirus & \\
\hline & Genus Ipomovirus & \\
\hline & Genus Torradovirus & \\
\hline \multirow[t]{4}{*}{ Aphis gossypii } & Cucumber mosaic virus (CMV) & \multirow[t]{2}{*}{ Pinto et al. (2008) } \\
\hline & Papaya ringspot virus (PRSV) & \\
\hline & Tobacco ringspot virus (TRSV) & Stace-Smith (1985) \\
\hline & Zucchini yellow mosaic virus (ZYMV) & Pinto et al. (2008) \\
\hline Epitrix hirtipennis & Tobacco ringspot virus (TRSV) & Stace-Smith (1985) \\
\hline \multirow[t]{3}{*}{ Liriomyza sativae } & Celery mosaic virus (CeMV) & \multirow[t]{3}{*}{ Zitter and Tsai (1977) } \\
\hline & Watermelon mosaic virus strain $1 * 1$ & \\
\hline & Watermelon mosaic virus strain 2 & \\
\hline \multirow[t]{2}{*}{ Tetranychus urticae } & Tobacco mosaic virus $(\mathrm{TMV})^{\star} 2$ & \multirow[t]{2}{*}{ Orlob (1968) } \\
\hline & Potato virus $X(\mathrm{PVX})^{\star 2}$ & \\
\hline
\end{tabular}


We found virus-like symptoms, showing mottle and deformation on pepino leaves. However, none of the tested viruses were detected in any plant. Our internet-based image searching results showed that the symptoms were similar to those on pepper or chili plants by Polyphagotarsonemus latus. Grinberg et al. (2005) described that young leaves were usually affected by $P$. latus and consequently showed distortion and leaf-curl downwards. This suggests that our symptomatic leaves might be caused by $P$. latus. Further research is required to reveal any effect of $P$. latus on pepino.

No related virus was detected in the present study, whereas two virus species had been detected from pepino plants in Japan: Alfalfa mosaic virus (AMV) and Cucumber mosaic virus (CMV) inducing chlorotic ring spots and mosaic symptoms on pepino plants, respectively (Honda et al. 1986). Moreover, Pepino mosaic virus (PepMV) was first isolated from pepinos showing yellow mosaic symptoms in Peru in 1974 (Jones et al. 1980). Currently, PepMV has become a major pathogen of tomato plants worldwide and is one of the quarantine pathogens strictly prohibited from entry into Japan. Pepino latent virus (PepLV; later reclassified as Potato virus $S$ (PVS)) was detected from pepino cuttings in New Zealand, even though pepino plants with no symptoms had been imported from Chile (Thomas et al. 1980). With the increase in pepino production in China, Potato virus $H$ (PVH) infected pepino, with no obvious symptoms (Abouelnasr et al. 2014) (Table 6). Pests and pathogens in many crops cause tremendous losses both in terms of quantity and quality. Monitoring, detection, and identification of pests and pathogens prior to the introduction of a new crop or during production are important to efficiently regulate their future damage. Our findings will aid in understanding the incidence of pests and viral diseases on pepino plants and developing better crop production systems to combat pests and diseases.

Table 6.

Viruses infecting pepino plants naturally around the world.

\begin{tabular}{|l|l|l|l|l|l|l|}
\hline Family & Genus & Species & Acronym & $\begin{array}{l}\text { Symptoms on } \\
\text { pepino }\end{array}$ & First reported & Reference \\
country
\end{tabular}




\section{Acknowledgements}

We are highly grateful to all the members of our project for kindly offering materials, supporting our field investigations and/or providing valuable comments on the manuscript. We also thank the members of the Laboratory of Vegetables and the Laboratory of Plant Pathology for their committed cultivation and management of pepino plants in our experimental fields. We are also indebted to Jenő Kontschán (Hungarian Academy of Sciences, Hungary) and Alina Avanesyan (Grand View University, USA) for their critical reading of the manuscript and for giving valuable comments. This study was financially supported by the Strategic Research Project from Tokyo University of Agriculture, Tokyo, Japan. We would like to thank Editage (www.editage.jp) for English language editing.

\section{References}

- $\quad$ Abouelnasr H, Li Y, Zhang Z, Liu J, Li S, Li D, Yu J, McBeath JH, Han C (2014) First report of Potato virus H on Solanum muricatum in China. Plant Disease 98: 1016. https://doi.org/10.1094/PDIS-01-14-0024-PDN

- $\quad$ Akyazi R (2012) First report of Aculops lycopersici (Tryon, 1917) (Acari: Eriophyidae) on Pepino in Turkey. Journal of Entomological and Acarological Research 2012 44: e20. https://doi.org/10.4081/jear.2012.e20

- $\quad$ Badge J, Brunt A, Carson R, Dagless E, Karamagioli M, Phillips S, Seal S, Turner R, Foster GD (1996) A carlavirus-specific PCR primer and partial nucleotide sequence provides further evidence for the recognition of cowpea mild mottle virus as a whiteflytransmitted carlavirus. European Journal of Plant Pathology 102: 305-310. https:// doi.org/10.1007/BF01877970

- $\quad$ Choi SK, Choi JK, Park WM, Ryu KH (1999) RT-PCR detection and identification of three species of cucumoviruses with a genus-specific single pair of primers. Journal of Virological Methods 83: 67-73. https://doi.org/10.1016/S0166-0934(99)00106-8

- Dolby CA, Jones RAC (1988) The relationship between the Andean strain of potato virus $S$ and pepino latent virus. Annals of Applied Biology 112: 231-234. https:// doi.org/10.1111/j.1744-7348.1988.tb02058.x

- $\quad$ Ehara S, Gotoh T (Eds) (2009) Colored Guide to the Plant Mites of Japan. Zenkoku Noson Kyoiku Kyokai, Tokyo, vi+349 pp. [In Japanese]. [ISBN 4-05-402370-3]

- $\quad$ Furukawa M (2005) Noctuidae. In: Ishiwata S, Hanada S, Hayashi F, Yamazaki T, Uemura Y, Aoki N, Hayashi M, Nozaki T, Fukuda H, Kishida Y, Hayashi N, Shinohara A, Shinonaga $T$ (Eds) Insect Larvae of Japan. Gakken, Tokyo, 217-225 pp. [In Japanese]. [ISBN 4-05-402370-3].

- Furusato K (1984) Atarashii-Yasai "Pepino" to sono-Tsukuri-kata [A new vegetable "pepino" and its cultivation]. Nogyo Gijutsu Kenkyu 38 (7): 36-37. [In Japanese].

- $\quad$ Furusato K (1986) Peru ni-okeru Pepino no Saibai-jôkyô [The situation in cultivation of pepino in Peru]. Nogyo Gijutsu Kenkyu 40 (6): 46-47. [In Japanese].

- Galbreath RA, Clearwater JR (1983) Pheromone monitoring of Sceliodes cordalis, a pest of pepino. In: Hartley M (Ed.) Proceedings of 36th New Zealand Weed and Pest Control Conference. Hastings, 9-11 August 1983. The New Zealand Weed and Pest Control Society Inc., Palmerston North, New Zealand, 128-130 pp. 
- $\quad$ Grinberg M, Perl-Treves R, Palevsky E, Shomer I, Soroker V (2005) Interaction between cucumber plants and the broad mite, Polyphagotarsonemus latus from damage to defense gene expression. Entomologia Experimentalis et Applicata 115: 135-144. https://doi.org/10.1111/j.1570-7458.2005.00275.x

- Ha C, Coombs S, Revill PA, Harding RM, Vu M, Dale JL (2008) Design and application of two novel degenerate primer pairs for the detection and complete genomic characterization of potyviruses. Archives of Virology 153: 25-36. https://doi.org/10.1007/ s00705-007-1053-7

- $\quad$ Harada H, Takizawa H (2012) Occurrence of Epitrix hirtipennis (Melsheimer) (Coleoptera: Chrysomelidae), an alien insect pest, in Japan. Japanese Journal of Applied Entomology and Zoology 56: 117-120. [In Japanese with English summary]. https://doi.org/10.1303/ijaez.2012.117

- Heiser CB (1964) Origin and variability of the pepino (Solanum muricatum): A preliminary report. Baileya 12: 151-158.

- Honda Y, Hanada K, Ushiyama K, Zenbayashi R, Tochihara H (1986) Alfalfa mosaic virus and cucumber mosaic virus isolated from pepino (Solanum muricatum). Annals of the Phytopathological Society of Japan 52: 870-873. [In Japanese with English abstract]. https://doi.org/10.3186/ijphytopath. 52.870

- Iwasaki A, Kasugai K, Iwaizumi R, Sasakawa M (2000) A newly recorded pest, Liriomyza sativae Blanchard in Japan. Plant Protection 54 (4): 142-147. [In Japanese].

- Japan Meteorological Agency (2017) http://www.jma.go.jp/jma/index.html. Accessed on: 2017-3-29.

- Jones RAC, Koenig R, Lesemann D (1980) Pepino mosaic virus, a new potexvirus from pepino (Solanum muricatum). Annals of Applied Biology 94: 61-68. https:// doi.org/10.1111/j.1744-7348.1980.tb03896.x

- $\quad$ Kato K, Onuki M, Fuji S, Hanada K (1998) The first occurrence of tomato yellow leaf curl virus in tomato (Lycopersicon esculentum Mill.) in Japan. Annals of the Phytopathological Society of Japan 64: 552-559. [In Japanese]. https://doi.org/10.3186/ jjphytopath.64.552

- Kawai S (1980) Scale Insects of Japan in Colors. Zenkoku Noson Kyoiku Kyokai, Tokyo, viii+455 pp. [In Japanese]. [ISBN 4-88137-011-1]

- $\quad$ Kita N (1986) Pepino no Saibai-gijutsu [Cultivation technique of pepino]. Yasai Engei Gijutsu 13 (9): 6-10. [In Japanese].

- Larraín SP (2002) Incidencia de insectos y ácaros plagas en pepino dulce (Solanum muricatum Ait.) culticado en la IV Región. Agricultura Técnica 61: 15-26. [In Spanish]. https://doi.org/10.4067/S036528072002000100002

- $\quad$ Masumoto M, Okajima S (2013) Review of the genus Thrips and related genera (Thysanoptera, Thripidae) from Japan. Zootaxa 3678: 1-65. https://doi.org/10.11646/ zootaxa.3678.1.1

- $\quad$ Matsumoto K (2004) Hemiptera (Sternorrhyncha: Psylloidea \& Aleyrodoidea). In: Kanagawa Konchû Danwakai Insect Fauna of Kanagawa, I (General Remarks, Protura, Diplura, Collembola, Archaeognatha, Thysanura, Ephemeroptera, Odonata, Plecoptera, Grylloblattodea, Orthoptera, Phasmida, Dermaptera, Mantodea, Blattaria, Isoptera, Psocoptera, Mallophaga, Anoplura, Hemiptera). Kanagawa Konchû Danwakai, Odawara, 273-282 pp. [In Japanese]. [ISBN 4-9902315-1-1].

- Matsumoto Y (2008) A Guide Illustrated Book of Aphids. Zenkoku Noson Kyoiku Kyokai, Tokyo, (10)+239 pp. [In Japanese]. [ISBN 978-4-88137-134-3] 
- Matsuura S, Hoshino S (2008) Comparative spatial dispersal of Tomato yellow leaf curl virus vectored by $B$ and $Q$ biotypes of Bemisia tabaci in tomato glasshouses.

Phytoparasitica 36: 42-51. https://doi.org/10.1007/BF02980747

- Moriones E, Navas-Castillo J (2000) Tomato yellow leaf curl virus, an emerging virus complex causing epidemics worldwide. Virus Research 71: 123-134. https:// doi.org/10.1016/S0168-1702(00)00193-3

- $\quad$ Moritsu M (1983) Aphids of Japan in Colors. Zenkoku Noson Kyoiku Kyokai, Tokyo, 545 pp. [In Japanese]. [ISBN 4-88137-017-0]

- Nakajima H, Yamamoto M (2004) Lepidoptera (excl. Hesperioidea \& Papilionoidea). In: Kanagawa Konchû Danwakai Insect Fauna of Kanagawa, III (Mecoptera, Siphonaptera, Diptera, Lepidoptera, Trichoptera, Hymenoptera). Kanagawa Konchû Danwakai, Odawara, 907-1158 pp. [In Japanese]. [ISBN 4-9902315-1-1].

- Navas-Castillo J, Fiallo-Olivé E, Sánchez-Campos S (2011) Emerging virus diseases transmitted by whiteflies. Annual Review of Phytopathology 49: 219-248. https:// doi.org/10.1146/annurev-phyto-072910-095235

- $\quad$ Odagiri T, Nishiyama T, Inaba K, Fukuoka N, Saida Y (1986) Cultivation management and plant physiology and ecology. 12. Establishment of cultivation system for pepino. Sosai Shiken Seisekisho 1986: 120-122. [In Japanese].

- Ohnishi J, Yamaguchi H, Saito A (2016) Analysis of the Mild strain of tomato yellow leaf curl virus, which overcomes $T y-2$ gene-mediated resistance in tomato line H24. Archives of Virology 161: 2207-2217. https://doi.org/10.1007/s00705-016-2898-4

- Orlob GB (1968) Relationship between Tetranychus urticae Koch and some plant viruses. Virology 35: 121-133. https://doi.org/10.1016/0042-6822(68)90312-7

- Ozawa A (1986) Pepino no Seitaiteki-tokusei to Saibaijô no Ryûi-ten [Ecological properties and points of attention for cultivation in pepino]. Shisetsu Engei 28 (1): 26-29. [In Japanese].

- $\quad$ Pinto ZV, Rezende JAM, Yuki VA, Piedade SMS (2008) Ability of Aphis gossypii and Myzus persicae to transmit Cucumber mosaic virusin single and mixed infection with two potyviruses to zucchini squash. Summa Phytopathologica Summa Phytopathologica 34: 183-185. https://doi.org/10.1590/S0100-54052008000200016

- $\quad$ Purcifull D, Hiebert E, Edwardson J (1984) Description of Plant Viruses. No. 293.Watermelon mosaic virus 2. http://www.dpvweb.net/dpv/showdpv.php?dpvno=293. Accessed on: 2017-6-29.

- $\quad$ Riley DG, Joseph SV, Srinivasan R, Diffie S (2011) Thrips vectors of tospoviruses. Journal of Integrated Pest Management 1 (2): 1-10. https://doi.org/10.1603/IPM10020

- Sakata Y (2011) Pepino. In: Nosangyoson-bunkakyokai Encyclopedia of growing vegetable 20. 2nd edition. Nosangyoson-bunkakyokai, Tokyo, 305-309 pp. [In Japanese].

- $\quad$ Stace-Smith R (1985) Description of Plant Viruses. No. 309. Tobacco ringspot virus . http://www.dpvweb.net/dpv/showdpv.php?dpvno=309. Accessed on: 2017-6-29.

- Takagi K (1985) Kasai Pepino [Pepino, a fruit vegetable]. Garden Life 3: 67-70. [In Japanese].

- Takahashi M (1985) Pepino. In: Kiyota I (Ed.) Yûbô-shin-yasai no Tsukuri-kata [Cultivation methods for new prospective vegetables]. Rural Culture Association Japan, Tokyo, 112-120 pp. [In Japanese]. [ISBN 4-540-85003-2]. 
- Takahashi M (1986) Shin-dônyû-kasai Pepino no Tokusei to Riyô-hô [Property and usage in pepino, a newly introduced vegetable]. Kongetsu no Noyaku 30 (1): 42-46. [In Japanese].

- Takahata K (2017) Effects of stem constriction using steel washer rings on the soluble solids content of pepino (Solanum muricatum Ait.) fruit. The Horticulture Journal Preview https://doi.org/10.2503/horti.OKD-034

- $\quad$ Tanaka H, Uesato T (2012) New records of some potential pest mealybugs (Hemiptera: Coccoidea: Pseudococcidae) in Japan. Applied Entomology and Zoology 47: 413-419. https://doi.org/10.1007/s13355-012-0134-6

- The Japanese Society of Applied Entomology and Zoology (2006) Major Insect and Other Pests of Economic Plants in Japan. Revised Edition. The Japanese Society of Applied Entomology and Zoology, Tokyo, v+387 pp.

- $\quad$ Thomas W, Mohamed NA, Fry ME (1980) Properties of a carlavirus causing a latent infection of pepino (Solanum muricatum). Annals of Applied Biology 95: 191-196. https://doi.org/10.1111/j.1744-7348.1980.tb04738.x

- Umeya K, Okada T (Eds) (2003) Agricultural Insect Pests in Japan. Zenkoku Noson Kyoiku Kyokai, Tokyo, 1203 pp. [In Japanese]. [ISBN 4-88137-103-7]

- van der Vlugt RAA, Berendsen M (2002) Development of a general potexvirus detection method. European Journal of Plant Pathology 108: 367-371. https://doi.org/10.1023/ A:1015644409484

- $\quad \mathrm{Xu} \mathrm{H}, \mathrm{Nie} \mathrm{J}$ (2006) Identification, characterization, and molecular detection of Alfalfa mosaic virus in Potato. Phytopathology 96: 1237-1242. https://doi.org/10.1094/ PHYTO-96-1237

- $\quad$ Yasunaga T, Schuh RT, Duwal RK (2015) Taxonomic review of the plant bug genus Campylomma Reuter from Japan (Heteroptera: Miridae: Phylinae: Nasocorini), with descriptions of two new species. Tijdschrift voor Entomologie 158: 49-69. https:// doi.org/10.1163/22119434-00002046

- $\quad$ Yasunaga T, Takai M, Kawasawa T (Eds) (2001) A Field Guide to Japanese Bugs Terrestrial Heteropterans. Vol. 2. Zenkoku Noson Kyoiku Kyokai, Tokyo, 350 pp. [In Japanese]. [ISBN 4-88137-089-8]

- $\quad$ Zitter TA, Tsai JH (1977) Transmission of three potyviruses by the leaf miner, Liriomyza sativae (Dipterta: Agromyzidae). Plant Disease Reporter 61: 1025-1029.

\section{Endnotes}

*1 Reclassified as PRSV (Purcifull et al. 1984)

*2 Experimentally transmissible

*3 Reclassified as an isolate of Potato virus $S$ (PVS) based on biological and serological characteristics (Dolby and Jones 1988). 